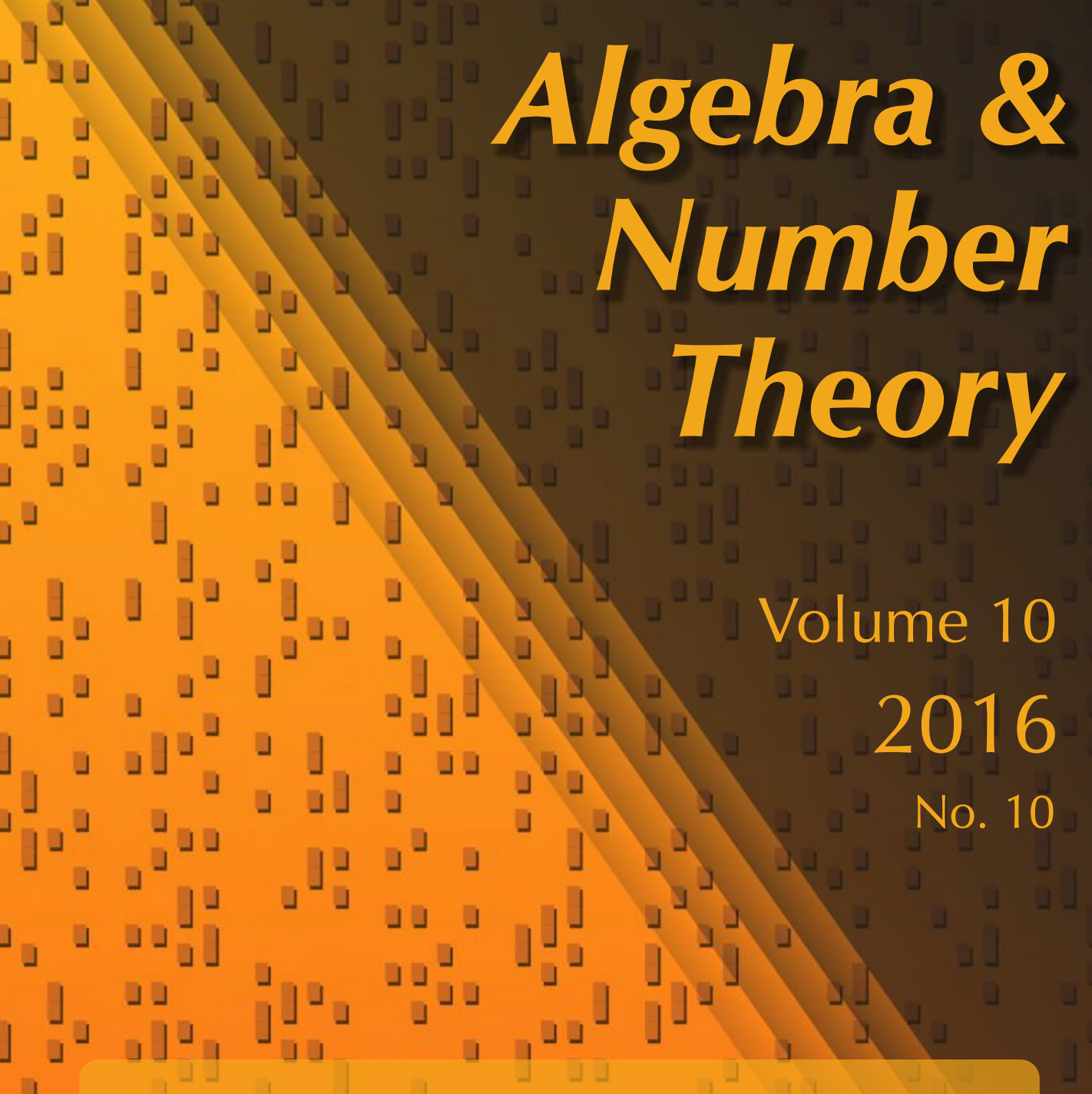

Every integer greater than 454 is the sum of at most seven positive cubes

Samir Siksek

J.

ل 


\title{
Every integer greater than 454 is the sum of at most seven positive cubes
}

\author{
Samir Siksek
}

A long-standing conjecture states that every positive integer other than

$15,22,23,50,114,167,175,186,212$,

$231,238,239,303,364,420,428,454$

is a sum of at most seven positive cubes. This was first observed by Jacobi in 1851 on the basis of extensive calculations performed by the famous computationalist Zacharias Dase. We complete the proof of this conjecture, building on previous work of Linnik, Watson, McCurley, Ramaré, Boklan, Elkies, and many others.

\section{Historical introduction}

In 1770, Edward Waring stated in his Meditationes Algebraica,

Omnis integer numerus vel est cubus, vel e duobus, tribus, 4, 5, 6, 7, 8, vel novem cubis compositus, ...

Waring's assertion can be concisely reformulated as the assertion that "every positive integer is the sum of nine nonnegative cubes". Henceforth, by a cube we shall mean a nonnegative cube. In the 19th century, numerical experimentation led to refinements of Waring's assertion for sums of cubes. As noted by Dickson [1927], "At the request of Jacobi, the famous computer Dase constructed a table showing the least number of positive cubes whose sum is any $p<12000$ ”. In an influential Crelle paper, Jacobi [1851] made a series of observations based on Dase's table: every positive integer other than 23 and 239 is the sum of eight cubes, every integer greater than 454 is the sum of seven cubes, and every integer greater than 8042 is the sum of six cubes. Jacobi believed that every sufficiently large integer is the sum of five cubes, whilst recognizing that the cutoff point must be far beyond Dase's table, and he wondered if the same is true for sums of four cubes. He noted that integers equivalent to $4,5(\bmod 9)$ cannot be sums of three cubes. Later

The author is supported by an EPSRC Leadership Fellowship EP/G007268/1, and EPSRC LMF: L-Functions and Modular Forms Programme Grant EP/K034383/1.

MSC2010: 11P05.

Keywords: Waring, cubes, sums of cubes. 
computations by Romani [1982] convincingly suggest that every integer greater than 1290740 is the sum of five cubes, and by Deshouillers et al. [2000] that every integer greater than 7373170279850 is the sum of four cubes.

Progress towards proving these observations of Waring, Jacobi and others has been exceedingly slow. Maillet [1895] showed that twenty-one cubes are enough to represent every positive integer. At the heart of Maillet's proof is an idea crucial to virtually all future developments; the identity $(r+x)^{3}+(r-x)^{3}=2 r^{3}+6 r x^{2}$ allows one to reformulate the problem of representing an integer as the sum of a (certain number of) cubes in terms of representing a related integer as the sum of (a smaller number of) squares. Exploiting this idea, Wieferich [1908] proved Waring's assertion (Wieferich's proof had a mistake that was corrected by Kempner [1912]). In fact, the theoretical part of Wieferich's proof showed that all integers exceeding $2.25 \times 10^{9}$ are sums of nine cubes. Completing the proof required appealing to a table of von Sterneck [1903] (who extended Dase's table to 40000 ), and applying what is now known as the greedy algorithm to reach the bound.

Soon thereafter, Landau [1908] showed that every sufficiently large integer is the sum of eight cubes. This was made effective by Baer [1913], who showed that every integer greater than or equal to $14.1 \times 233^{6} \approx 2.26 \times 10^{15}$ is the sum of eight cubes. Dickson [1939] completed the proof of Jacobi's observation that all positive integers other than 23 and 239 are sums of eight cubes. Remarkably, Dickson's proof relied on extending von Sterneck's table to 123000 (with the help of his assistant, Miss Evelyn Garbe) and then applying the greedy algorithm to reach Baer's bound.

Linnik [1943] showed that every sufficiently large integer is the sum of seven cubes. A substantially simpler proof (though still ineffective) was given by Watson [1951]. Linnik's seven cubes theorem was first made effective by McCurley [1984], who showed that it is true for integers greater than $\exp (\exp (13.94))$. Ramaré [2005] improved this to $\exp (205000)$ and finally to $\exp (524) \approx 3.72 \times 10^{227}$ [Ramaré 2007]. This bound is way beyond computer searches combined with the greedy algorithm. In [Deshouillers et al. 2000], it is shown that every integer between 1290741 and $10^{16}$ is a sum of five cubes. As observed in [Ramaré 2007], combining this with the greedy algorithm [Bertault et al. 1999, Lemma 3], we can easily deduce that every integer $455 \leq N \leq \exp (78.7) \approx 1.51 \times 10^{34}$ is the sum of seven cubes.

There has been a number of partial results concerning sums of seven cubes. Bertault et al. [1999] show that every nonnegative integer which is a cubic residue modulo 9 and an invertible cubic residue modulo 37 is a sum of 7 cubes. Boklan and Elkies [2009] show that every multiple of 4 greater than 454 is the sum of seven cubes, whilst Elkies [2010] shows the same for integers equivalent to $2(\bmod 4)$.

In this paper we complete the proof of Jacobi's seven cubes conjecture, building on the aforementioned great works. 


\section{Theorem 1. Every positive integer other than}

$15,22,23,50,114,167,175,186,212,231,238,239,303,364,420,428,454$ is the sum of seven cubes.

An online supplement contains Magma scripts implementing the algorithms that support the proof.

\section{The main criterion}

Let $\mathscr{K}=\exp (524)$ and $\mathscr{K}^{\prime}=\exp (78.7)$. By results found in [Ramaré 2007] and [Deshouillers et al. 2000], it is sufficient to prove that every integer $\mathscr{K}^{\prime} \leq N \leq \mathscr{K}$ is the sum of seven cubes.

Results from [Boklan and Elkies 2009] and [Elkies 2010] allow us to restrict ourselves to odd integers $N$ (our method can certainly be adapted to deal with even integers, but restricting ourselves to odd integers brings coherence to our exposition). In this section we give a criterion (Proposition 2.2) for all odd integers $N$ in a range $K_{1} \leq N \leq K_{2}$ to be sums of seven cubes. Most of the remainder of the paper is devoted to showing that this criterion holds for each of the ranges $\left(\frac{9}{10}\right)^{n+1} \mathscr{K} \leq N \leq\left(\frac{9}{10}\right)^{n} \mathscr{K}$ with $0 \leq n \leq 4226$. This will complete the proof of Theorem 1 as

$$
\left(\frac{9}{10}\right)^{4227} \mathscr{K} \approx 1.42 \times 10^{34} \text { and } \mathscr{K}^{\prime} \approx 1.51 \times 10^{34} .
$$

Theorem 2 (Gauss, Legendre). Let $k \geq 0$ be an even integer. There exist integers $x, y, z$ such that

$$
x^{2}+x+y^{2}+y+z^{2}+z=k .
$$

Proof. Dividing by 2 we see that this is in fact the famous theorem, due to Gauss, that every nonnegative integer is the sum of three triangular numbers. Alternatively, we can rewrite (1) as

$$
(2 x+1)^{2}+(2 y+1)^{2}+(2 z+1)^{2}=4 k+3 .
$$

As $k$ is even, $4 k+3 \equiv 3(\bmod 8)$; by a theorem of Legendre, every positive integer equivalent to $3(\bmod 8)$ is the sum of three odd squares.

Throughout this section $m$ will denote a positive integer satisfying the conditions

(i) $m$ is a squarefree,

(ii) $3 \mid m$,

(iii) every prime divisor of $m / 3$ is $\equiv 5(\bmod 6)$.

Observe that $m \equiv 3(\bmod 6)$. Moreover, for any integer $N$, there is a unique integer $t \in[0, m)$ such that $N \equiv 8 t^{3}(\bmod m)$. Our starting point is a modified version of Lemma 3 of [Watson 1951]. 
Lemma 2.1. Let $0<K_{1}<K_{2}$ be real numbers. Let $m$ be a positive integer satisfying (i)-(iii) above. Let $\varepsilon_{m}$ and $\delta_{m}$ be real numbers satisfying

(iv) $0 \leq \varepsilon_{m}<\delta_{m} \leq 1$,

(v) $K_{1} \geq\left(8 \delta_{m}^{3}+\frac{1}{36}\right) m^{3}+3 m / 4$,

(vi) $K_{2} \leq\left(8 \varepsilon_{m}^{3}+\frac{1}{18}\right) m^{3}+m / 2$.

Let $K_{1} \leq N \leq K_{2}$ be an odd integer. Suppose $N \equiv 8 t^{3}(\bmod m)$ with $t \in\left[\varepsilon_{m} \cdot m, \delta_{m} \cdot m\right)$. Then $N$ is the sum of seven nonnegative cubes.

Proof. Write $m=6 r+3$. Let

$$
k=\frac{N-8 t^{3}}{m}-\left(r^{2}+r+1\right) .
$$

The quantity $k$ is an integer as $N \equiv 8 t^{3}(\bmod m)$, and even as $\left(N-8 t^{3}\right) / m$ and $r^{2}+r+1$ are both odd. Observe that

$$
\begin{aligned}
k & >\frac{N-8 \delta_{m}^{3} \cdot m^{3}}{m}-\left(r^{2}+r+1\right) & & \left(\text { since } t<\delta_{m} \cdot m\right) \\
& \geq \frac{K_{1}-8 \delta_{m}^{3} \cdot m^{3}}{m}-\left(r^{2}+r+1\right) & & \left(\text { since } N \geq K_{1}\right) \\
& =\frac{K_{1}-8 \delta_{m}^{3} \cdot m^{3}}{m}-\frac{m^{2}}{36}-\frac{3}{4} & & (\text { substituting } r=(m-3) / 6) \\
& \geq 0 & & (\text { by }(\mathrm{v})) .
\end{aligned}
$$

As $k$ is nonnegative and even, by the Gauss-Legendre theorem, there exist integers $x, y, z$ satisfying (1). We shall make use of the identity

$$
\begin{array}{r}
(r+1+x)^{3}+(r-x)^{3}+(r+1+y)^{3}+(r-y)^{3}+(r+1+z)^{3}+(r-z)^{3} \\
=(6 r+3)\left(r^{2}+r+1+x^{2}+x+y^{2}+y+z^{2}+z\right) .
\end{array}
$$

From the definition of $k$ in (3) and the fact that $m=6 r+3$, we see that $N-8 t^{3}$ is equal to the right-hand side of the identity (4). Hence

$$
N=(r+1+x)^{3}+(r-x)^{3}+(r+1+y)^{3}+(r-y)^{3}+(r+1+z)^{3}+(r-z)^{3}+(2 t)^{3} .
$$

To complete the proof it is enough to show that these cubes are nonnegative, or equivalently that

$$
-r-1 \leq x, y, z \leq r .
$$

This is equivalent to showing that

$$
-(2 r+1) \leq 2 x+1,2 y+1,2 z+1 \leq 2 r+1 .
$$


Now $(2 y+1)^{2},(2 z+1)^{2} \geq 1$ and so from (2), we have $(2 x+1)^{2} \leq 4 k+1$. It is therefore enough to show that $4 k+1 \leq(2 r+1)^{2}$ or equivalently that $k \leq r^{2}+r$. The following inequalities complete the proof:

$$
\begin{aligned}
k-r^{2}-r & =\frac{N-8 t^{3}}{m}-\left(2 r^{2}+2 r+1\right) & & (\text { from (3)) } \\
& \leq \frac{N-8 \varepsilon_{m}^{3} \cdot m^{3}}{m}-\left(2 r^{2}+2 r+1\right) & & \left(\text { since } t \geq \varepsilon_{m} \cdot m\right) \\
& \leq \frac{K_{2}-8 \varepsilon_{m}^{3} \cdot m^{3}}{m}-\left(2 r^{2}+2 r+1\right) & & \left(\text { since } N \leq K_{2}\right) \\
& \leq \frac{K_{2}-8 \varepsilon_{m}^{3} \cdot m^{3}}{m}-\frac{m^{2}}{18}-\frac{1}{2} & & (\text { substituting } r=(m-3) / 6) \\
& \leq 0 & & (\text { by (vi) }) .
\end{aligned}
$$

This simpleminded lemma has one serious flaw. The inequality $K_{1}<K_{2}$ and the conditions (iv)-(vi) together imply that

$$
\delta_{m}^{3}<\varepsilon_{m}^{3}+\frac{1}{288}-\frac{1}{32 m^{2}} .
$$

In particular, this forces the interval $\left[\varepsilon_{m} \cdot m, \delta_{m} \cdot m\right)$ to have length less than $m / \sqrt[3]{288} \approx 0.15 m$. On the other hand, the integer $t$ appearing in the lemma (which is the cube root of $N / 8$ modulo $m$ ) can be any integer in the interval $[0, m)$. Thus the lemma only treats a small fraction of the odd integers $K_{1} \leq N \leq K_{2}$. Our key innovation over the works mentioned in the introduction is to use not just one value of $m$, but many of them simultaneously. Each value of $m$ will give some information about those odd integers $K_{1} \leq N \leq K_{2}$ that cannot be expressed as sums of seven cubes; collecting this information will allow us to deduce a contradiction.

Let $x$ be a real number and $m$ be a positive integer. Define the quotient and remainder obtained on dividing $x$ by $m$ as

$$
\mathrm{Q}(x, m)=\lfloor x / m\rfloor, \quad \mathrm{R}(x, m)=x-\mathrm{Q}(x, m) \cdot m .
$$

In particular, $\mathrm{R}(x, m)$ belongs to the half-open interval $[0, m)$. If $x \in \mathbb{Z}$ then $\mathrm{R}(x, m)$ is the usual remainder on dividing by $m$ and $x \equiv \mathrm{R}(x, m)(\bmod m)$. Let $\varepsilon_{m}$ and $\delta_{m}$ be real numbers satisfying $0 \leq \varepsilon_{m}<\delta_{m} \leq 1$. Define

$$
\begin{aligned}
\operatorname{Bad}\left(m, \varepsilon_{m}, \delta_{m}\right) & =\left\{x \in \mathbb{R}: \mathrm{R}(x, m) \in[0, m) \backslash\left[\varepsilon_{m} \cdot m, \delta_{m} \cdot m\right)\right\} \\
& =\bigcup_{k=-\infty}^{\infty} k m+\left([0, m) \backslash\left[\varepsilon_{m} \cdot m, \delta_{m} \cdot m\right)\right) .
\end{aligned}
$$

The reader will observe, in Lemma 2.1, if $N$ is not the sum of seven cubes, then $t \in \operatorname{Bad}\left(m, \varepsilon_{m}, \delta_{m}\right)$, which explains our choice of the epithet "bad". Given a set of 
positive integers $\mathcal{W}$, and sequences $\underset{\sim}{\varepsilon}=\left(\varepsilon_{m}\right)_{m \in \mathcal{W}} \underset{\sim}{\delta}=\left(\delta_{m}\right)_{m \in \mathcal{W}}$ of real numbers satisfying $0 \leq \varepsilon_{m}<\delta_{m} \leq 1$ for all $m \in \mathcal{W}$, we define

$$
\operatorname{Bad}(\mathcal{W}, \underset{\sim}{\varepsilon}, \underset{\sim}{\delta})=\bigcap_{m \in \mathcal{W}} \operatorname{Bad}\left(m, \varepsilon_{m}, \delta_{m}\right)
$$

To make the notation less cumbersome, we usually regard the values $\varepsilon_{m}$ and $\delta_{m}$ as implicit, and write $\operatorname{Bad}(m)$ for $\operatorname{Bad}(m, \varepsilon, \delta)$, and $\operatorname{Bad}(\mathcal{W})$ for $\operatorname{Bad}(\mathcal{W}, \underset{\sim}{\varepsilon}, \underset{\sim}{\delta})$.

Proposition 2.2. Let $0<K_{1}<K_{2}$ be real numbers. Let $\mathcal{W}$ be a nonempty finite set of integers such that every element $m \in \mathcal{W}$ satisfies conditions (i)-(iii). Suppose moreover, that for each $m \in \mathcal{W}$, there are real numbers $\varepsilon_{m}$ and $\delta_{m}$ satisfying conditions $(i v)-(v i)$. Let $M=\operatorname{lcm}(\mathcal{W})$. Let $\mathfrak{S} \subset[0,1]$ be a finite set of rational numbers $a / q$ (here $\operatorname{gcd}(a, q)=1)$ with denominators $q$ bounded by $\sqrt[3]{M / 2 K_{2}}$. Suppose that

$$
\operatorname{Bad}(\mathcal{W}) \cap[0, M) \subseteq \bigcup_{a / q \in \mathfrak{S}}\left(\frac{a}{q} M-\frac{\sqrt[3]{M / 16}}{q}, \frac{a}{q} M+\frac{\sqrt[3]{M / 16}}{q}\right)
$$

Then every odd integer $K_{1} \leq N \leq K_{2}$ is the sum of seven nonnegative cubes.

Proof. Let $N$ be an odd integer satisfying $K_{1} \leq N \leq K_{2}$. It follows from assumptions (i)-(iii) that $M=\operatorname{lcm}(\mathcal{W})$ is squarefree and divisible only by 3 and primes equivalent to 5 (mod 6). Thus there exists a unique integer $T \in[0, M)$ such that

$$
N \equiv 8 T^{3}(\bmod M) \text {. }
$$

Suppose $N$ is not the sum of seven cubes. Then, by Lemma 2.1, for each $m \in \mathcal{W}$, we have $\mathrm{R}(T, m) \in[0, m) \backslash\left[\varepsilon_{m} \cdot m, \delta_{m} \cdot m\right)$. Thus $T \in \operatorname{Bad}(\mathcal{W}) \cap[0, M)$. By (7) there is some rational $a / q \in \mathfrak{S}$ such that

$$
-\frac{\sqrt[3]{M / 16}}{q}<T-\frac{a}{q} M<\frac{\sqrt[3]{M / 16}}{q}
$$

or equivalently

$$
-\frac{M}{2}<8(q T-a M)^{3}<\frac{M}{2} .
$$

Moreover, the denominator $q$ is bounded by $\sqrt[3]{M / 2 K_{2}}$ and so

$$
q^{3} N \leq \frac{M N}{2 K_{2}} \leq \frac{M}{2},
$$

as $N \leq K_{2}$. Hence

$$
\left|q^{3} N-8(q T-a M)^{3}\right|<M .
$$


However, by (8), we have $q^{3} N-8(q T-a M)^{3} \equiv 0(\bmod M)$. Thus $q^{3} N=$ $8(a T-a M)^{3}$. It follows that $N$ is a perfect cube, and so is certainly the sum of seven nonnegative cubes.

We shall mostly apply Proposition 2.2 with the parameter choices given by the following lemma.

Lemma 2.3. Let $K \geq 10^{5}$. Let $K_{1}=9 K / 10, K_{2}=K$. Let

$$
\frac{263}{100} K^{\frac{1}{3}} \leq m \leq \frac{292}{100} K^{\frac{1}{3}} .
$$

Then conditions (iv)-(vi) are satisfied with $\varepsilon_{m}=0$ and $\delta_{m}=\frac{1}{10}$.

\section{Plan for the paper}

The rest of the paper is devoted to understanding and computing the intersections $\operatorname{Bad}(\mathcal{W}) \cap[0, M)$ appearing in Proposition 2.2. Section 4 collects various properties of remainders and bad sets that are used throughout. Section 5 provides justification, under a plausible assumption, that the intersection $\operatorname{Bad}(\mathcal{W}) \cap[0, M)$ should be decomposable as in (7). Section 6 gives an algorithm (Algorithm 1) which takes as input a finite set of positive integers $\mathcal{W}$ and an interval $[A, B)$ and returns the intersection $\operatorname{Bad}(\mathcal{W}) \cap[A, B)$. We also give a heuristic analysis of the algorithm and its running time. Section 7 introduces the concept of a "tower", which is a sequence

$$
\mathcal{W}_{0} \subseteq \mathcal{W}_{1} \subseteq \mathcal{W}_{2} \subseteq \cdots \subseteq \mathcal{W}_{r}=\mathcal{W}
$$

Letting $M_{i}=\operatorname{lcm}\left(\mathcal{W}_{i}\right)$, we prove the recursive formula for computing $\operatorname{Bad}\left(\mathcal{W}_{i}\right) \cap$ $\left[0, M_{i}\right)$ in terms of $\operatorname{Bad}\left(\mathcal{W}_{i-1}\right) \cap\left[0, M_{i-1}\right)$. This recursive formula together with Algorithm 1 is the basis for a much more efficient algorithm (Algorithm 2) for computing $\operatorname{Bad}(\mathcal{W}) \cap[0, M)$ given in Section 7 .

In Section 8 we let $M^{*}$ be the product of all primes $p \leq 167$ that are equivalent to $5(\bmod 6)$, and

$$
\mathcal{W}^{*}=\left\{m \mid M^{*}: 265 \times 10^{9} \leq m \leq 290 \times 10^{9}\right\} .
$$

We use a tower and Algorithm 2 to compute $\operatorname{Bad}\left(\mathcal{W}^{*}\right) \cap\left[0, M^{*}\right)$. The actual computation consumed about 18,300 hours of CPU time.

Section 9 is devoted to proving Theorem 1 for $N \geq\left(\frac{9}{10}\right)^{3998} \cdot \mathscr{K} \approx 4.28 \times 10^{44}$, where $\mathscr{K}=\exp (524)$. The approach is to divide the interval $\left(\frac{9}{10}\right)^{3998} \mathscr{K} \leq N \leq \mathscr{K}$ into subintervals $\left(\frac{9}{10}\right)^{n+1} \mathscr{K} \leq N \leq\left(\frac{9}{10}\right)^{n} \mathscr{K}$ with $0 \leq n \leq 3997$, and apply Proposition 2.2 and Lemma 2.3 to prove that all odd integers in the interval $\left(\frac{9}{10}\right)^{n+1} \mathscr{K} \leq N \leq\left(\frac{9}{10}\right)^{n} \mathscr{K}$ are sums of seven nonnegative cubes. Indeed, we show that given $0 \leq n \leq 3997$, there is some suitable positive $\kappa$ such that the elements of $\mathcal{W}_{0}=\kappa \cdot \mathcal{W}^{*}$ satisfy conditions (i)-(iii) (with $K_{1}=\left(\frac{9}{10}\right)^{n+1} \mathscr{K}$ and 
$\left.K_{2}=\left(\frac{9}{10}\right)^{n} \mathscr{K}\right)$ and that moreover, $\operatorname{Bad}\left(\mathcal{W}_{0}\right)=\kappa \operatorname{Bad}\left(\mathcal{W}^{*}\right)$. Thus the results of the huge computation of Section 8 are recycled 3998 times; on top of this $\mathcal{W}_{0}$ we construct a tower and continue until we have found a set $\mathcal{W}$ that satisfies the hypotheses of Proposition 2.2, thereby proving Theorem 1 for $N \geq\left(\frac{9}{10}\right)^{3998} \cdot \mathscr{K}$. The CPU time for the computations described in Section 9 was around 10,000 hours.

The proof of Theorem 1 is completed in Section 10 where a modified strategy is needed to handle the "small" ranges $\left(\frac{9}{10}\right)^{n+1} \mathscr{K} \leq N \leq\left(\frac{9}{10}\right)^{n} \mathscr{K}$ with $3998 \leq$ $n \leq 4226$. Although these intervals are small (and few) compared to those handled in Section 9, we are unable to recycle the computation of Section 8. This makes the computations far less efficient, though still practical. The CPU time for the computations described in Section 10 was around 2,750 hours.

\section{Some properties of remainders and bad sets}

Lemma 4.1. Let $m$ and $\kappa$ be positive integers with $\kappa \mid m$. Then for any real $x$ we have

$$
\mathrm{Q}\left(\frac{x}{\kappa}, \frac{m}{\kappa}\right)=\mathrm{Q}(x, m), \quad \mathrm{R}\left(\frac{x}{\kappa}, \frac{m}{\kappa}\right)=\frac{1}{\kappa} \mathrm{R}(x, m) .
$$

Let $\kappa$ be a positive integer. For a set $X \subset \mathbb{R}$ we denote $\kappa X=\{\kappa x: x \in X\}$.

Lemma 4.2. Let $m$ and $\kappa$ be positive integers. Let $0 \leq \varepsilon<\delta \leq 1$ be real numbers. Then

$$
\operatorname{Bad}(\kappa m, \varepsilon, \delta)=\kappa \cdot \operatorname{Bad}(m, \varepsilon, \delta) .
$$

Let $\mathcal{W}$ be a set of positive integers and for $m \in \mathcal{W}$ let $0 \leq \varepsilon_{m}<\delta_{m} \leq 1$ be real numbers. Let

$\mathcal{W}^{\prime}=\kappa \cdot \mathcal{W}, \quad \underset{\sim}{\varepsilon}=\left(\varepsilon_{m}\right)_{m \in \mathcal{W}}, \quad \underset{\sim}{\delta}=\left(\delta_{m}\right)_{m \in \mathcal{W}}, \quad{\underset{\sim}{\varepsilon}}^{\prime}=\left(\varepsilon_{m / \kappa}\right)_{m \in \mathcal{W}^{\prime}} \quad \stackrel{\sim}{\delta}^{\prime}=\left(\delta_{m / \kappa}\right)_{m \in \mathcal{W}^{\prime}}$

Then

$$
\operatorname{Bad}\left(\mathcal{W}^{\prime}, \underset{\sim}{\varepsilon^{\prime}}, \underset{\sim}{\delta^{\prime}}\right)=\kappa \cdot \operatorname{Bad}(\mathcal{W}, \underset{\sim}{\varepsilon}, \underset{\sim}{\delta})
$$

Proof. By (5) and Lemma 4.1,

$$
\begin{aligned}
x \in \operatorname{Bad}(\kappa m, \varepsilon, \delta) & \Longleftrightarrow \mathrm{R}(x, \kappa m) \in[0, \kappa m) \backslash[\varepsilon \cdot \kappa m, \delta \cdot \kappa m) \\
& \Longleftrightarrow 1 / \kappa \mathrm{R}(x, \kappa m) \in[0, m) \backslash[\varepsilon \cdot m, \delta \cdot m) \\
& \Longleftrightarrow \mathrm{R}(x / \kappa, m) \in[0, m) \backslash[\varepsilon \cdot m, \delta \cdot m) \\
& \Longleftrightarrow x / \kappa \in \operatorname{Bad}(m, \varepsilon, \delta) \\
& \Longleftrightarrow x \in \kappa \cdot \operatorname{Bad}(m, \varepsilon, \delta) .
\end{aligned}
$$

This proves the first part of the lemma. The second part now follows from (6). 
Lemma 4.3. Given positive integers $M_{1} \mid M_{2}$, we define the "natural" map

$$
\pi_{M_{2}, M_{1}}:\left[0, M_{2}\right) \longrightarrow\left[0, M_{1}\right), \quad x \mapsto \mathrm{R}\left(x, M_{1}\right) .
$$

Then $\pi_{M_{2}, M_{1}}$ is surjective, and for any $T \subseteq\left[0, M_{1}\right)$,

$$
\pi_{M_{2}, M_{1}}^{-1}(T)=\bigcup_{k=0}^{\left(M_{2} / M_{1}\right)-1}\left(k \cdot M_{1}+T\right)
$$

Lemma 4.4. Let $\mathcal{W}_{1}, \mathcal{W}_{2}$ be sets of positive integers with $\mathcal{W}_{1} \subseteq \mathcal{W}_{2}, M_{i}=\operatorname{lcm}\left(\mathcal{W}_{i}\right)$, and $\pi=\pi_{M_{2}, M_{1}}$. Write $\mathcal{U}=\mathcal{W}_{2} \backslash \mathcal{W}_{1}$. Then $\pi\left(\operatorname{Bad}\left(\mathcal{W}_{2}\right)\right) \subseteq \operatorname{Bad}\left(\mathcal{W}_{1}\right)$ and

$$
\operatorname{Bad}\left(\mathcal{W}_{2}\right) \cap\left[0, M_{2}\right)=\pi^{-1}\left(\operatorname{Bad}\left(\mathcal{W}_{1}\right) \cap\left[0, M_{1}\right)\right) \cap \operatorname{Bad}(\mathcal{U}) .
$$

Proof. Let $x \in \mathbb{R}$ and let $y=\pi(x)=\mathrm{R}\left(x, M_{1}\right)$. If $m \in \mathcal{W}_{1}$ then $\mathrm{R}(y, m)=\mathrm{R}(x, m)$, as $m \mid M_{1}$. Observe that

$$
\begin{aligned}
x \in \operatorname{Bad}\left(\mathcal{W}_{2}\right) & \Longleftrightarrow \mathrm{R}(x, m) \notin\left[\varepsilon_{m} \cdot m, \delta_{m} \cdot m\right) \text { for all } m \in \mathcal{W}_{2} \\
& \Longrightarrow \mathrm{R}(x, m) \notin\left[\varepsilon_{m} \cdot m, \delta_{m} \cdot m\right) \text { for all } m \in \mathcal{W}_{1} \\
& \Longleftrightarrow \mathrm{R}(y, m) \notin\left[\varepsilon_{m} \cdot m, \delta_{m} \cdot m\right) \text { for all } m \in \mathcal{W}_{1} \\
& \Longleftrightarrow y \in \operatorname{Bad}\left(\mathcal{W}_{1}\right) .
\end{aligned}
$$

This shows that $\pi\left(\operatorname{Bad}\left(\mathcal{W}_{2}\right)\right) \subseteq \operatorname{Bad}\left(\mathcal{W}_{1}\right)$. The rest of the lemma easily follows.

\section{Gaps and ripples}

We will soon give an algorithm for computing the intersection

$$
\operatorname{Bad}(\mathcal{W}) \cap[0, M)=\left(\bigcap_{m \in \mathcal{W}} \operatorname{Bad}(m)\right) \cap[0, M), \quad M=\operatorname{lcm}(\mathcal{W}),
$$

given a set $\mathcal{W}$ that satisfies the conditions of Proposition 2.2. The statement of Proposition 2.2 (notably (7)) suggests that we are expecting this intersection to be concentrated in small intervals around $a M / q$ for certain $a / q$ with relatively small denominators $q$. In this section we provide an explanation for this. The situation is easier to analyze if we make choices of parameters as in Lemma 2.3. Thus for this section we fix the choices $\varepsilon_{m}=0, \delta_{m}=\frac{1}{10}$, and hence $\operatorname{Bad}(m)=\operatorname{Bad}\left(m, 0, \frac{1}{10}\right)$. We suppose that the elements $m \in \mathcal{W}$ belong to an interval of the form

$$
\frac{263}{100} L \leq m \leq \frac{292}{100} L,
$$

for some $L>0$ (see Lemma 2.3). In fact, we show that if $q$ is large, and if the residues of the integers $a M / m$ are regularly distributed modulo $q$ (in a sense that will be made precise), then the intersection $\operatorname{Bad}(\mathcal{W}) \cap[0, M)$ contains no points in a certain explicitly given neighborhood of $a M / q$. Likewise we show, for certain $a / q$ 
with $q$ small, that $\operatorname{Bad}(\mathcal{W}) \cap[0, M)$ does contain some points near $a M / q$. We stress that the material in this section does not form part of our proof of Theorem 1 . It does however explain the results of our computations that do form part of the proof of Theorem 1, and it lends credibility to them.

We fix the following notation throughout this section:

- $L$ is a positive real number.

- $\mathcal{W}$ is a nonempty set of positive integers that belong to the interval (12).

- $M=\operatorname{lcm}(\mathcal{W})$.

\section{Ripples.}

Proposition 5.1. Suppose $M \geq 2000$ L. Let a $/ q \in[0,1)$ be a fraction in simplest form with $1 \leq q \leq 9$ and $0 \leq a \leq q-1$. For $0 \leq k \leq 9-q$ let

$$
\psi_{k}=\frac{292}{100}\left(\frac{k}{q}+\frac{1}{10}\right), \quad \Psi_{k}=\frac{263}{100} \cdot \frac{k+1}{q} .
$$

Then $\psi_{k}<\Psi_{k}$ and

$$
\bigcup_{k=0}^{9-q}\left(\frac{a}{q} M+\psi_{k} \cdot L, \frac{a}{q} M+\Psi_{k} \cdot L\right) \subseteq \operatorname{Bad}(\mathcal{W}) \cap[0, M) .
$$

This recipe gives 103 disjoint intervals contained in $\operatorname{Bad}(\mathcal{W}) \cap[0, M)$ of total length $\xi \cdot L$ where

$$
\xi=\frac{261707}{10500} \approx 24.9 .
$$

We shall informally refer to the union of intervals (14) as a ripple emanating from $a M / q$ in the positive direction. The reader will easily modify the proof below to show, under similar hypotheses, that there are ripples emanating from the $a \mathrm{M} / \mathrm{q}$ in the negative direction.

Proof. It is easy to check that $\psi_{k}<\Psi_{k}$ for $q \leq 9$ and $0 \leq k \leq 9-q$. The assumption $M \geq 2000 L$ ensures that the 103 intervals are contained in $[0, M)$ and are disjoint, so it is enough to show that the intervals are contained in $\operatorname{Bad}(\mathcal{W})$. Let $\alpha$ be a real number belonging to the interval $\psi_{k} \cdot L<\alpha<\Psi_{k} \cdot L$. We would like to show that $a M / q+\alpha \in \operatorname{Bad}(m)$ for all $m \in \mathcal{W}$. Let $m \in \mathcal{W}$. It follows from (12) and (13) that

$$
\left(\frac{k}{q}+\frac{1}{10}\right) m \leq \psi_{k} \cdot L<\alpha<\Psi_{k} \cdot L \leq \frac{k+1}{q} m .
$$

As $m \mid M$ we can write $a M=u m$ with $u \in \mathbb{Z}$. Now $u=b q+s$ where $0 \leq s \leq q-1$. Thus

$$
\frac{a}{q} M=b m+\frac{s}{q} m
$$


From (15),

$$
b m+\frac{k+s}{q} m+\frac{m}{10}<\frac{a}{q} M+\alpha<b m+\frac{k+s+1}{q} m .
$$

Let $k+s=q t+v$ where $0 \leq v \leq q-1$. Hence

$$
(b+t) m+\left(\frac{v}{q}+\frac{1}{10}\right) m<\frac{a}{q} M+\alpha<(b+t) m+\frac{v+1}{q} m .
$$

Observe that

$$
\frac{1}{10} \leq \frac{v}{q}+\frac{1}{10}<\frac{v+1}{q} \leq 1
$$

as $q \leq 9$ and $0 \leq v \leq q-1$. Thus $\mathrm{Q}(a M / q+\alpha, m)=b+t$ and

$$
\frac{m}{10}<\mathrm{R}\left(\frac{a M}{q}+\alpha, m\right)<m \text {. }
$$

This shows that $a M / q+\alpha \in \operatorname{Bad}(m)$ as required.

In the above proposition we showed the existence of ripples emanating from $a M / q$ for $q \leq 9$. There can also be ripples emanating for $a M / q$ for larger values of $q$ if the sequence of residues $\overline{a M / m}$ in $\mathbb{Z} / q \mathbb{Z}$ contains large gaps as illustrated by the following proposition.

Proposition 5.2. Let a $/ q \in(0,1)$ be a rational number in simplest form with $q \geq 11$ and $1 \leq a \leq q-1$. Let $(q-10) / 10<d<q-1$ be an integer, and let $s$ be $a$ nonnegative integer satisfying

$$
s<q-d-1, \quad s<\frac{263}{290}(10 d+10-q) .
$$

Suppose

$$
\overline{s+1}, \overline{s+2}, \ldots, \overline{s+d} \notin\{\overline{a M / m}: m \in \mathcal{W}\} \subseteq \mathbb{Z} / q \mathbb{Z}
$$

Let

$$
\pi=\frac{292}{100} \cdot \frac{s}{q}, \quad \Pi=\frac{263}{100}\left(\frac{s+d+1}{q}-\frac{1}{10}\right) .
$$

Then $\pi<\Pi$ and

$$
\left(\frac{a}{q} M-\Pi \cdot L, \frac{a}{q} M-\pi \cdot L\right) \subseteq \operatorname{Bad}(\mathcal{W})
$$

Proof. Let $m \in \mathcal{W}$, and recall that $m \mid M$. Thus $a M / m$ is an integer, and hence so is $\mathrm{R}(a M / m, q)$. By assumption (17),

$$
\mathrm{R}(a M / m, q) \neq s+1, s+2, \ldots, s+d .
$$

Thus $\mathrm{R}(a M / m, q) \notin(s, s+d+1)$. By Lemma 4.1,

$$
\mathrm{R}(a M / q, m)=\mathrm{R}(a M, q m) \cdot 1 / q=\mathrm{R}(a M / m, q) \cdot m / q .
$$


Thus

$$
\mathrm{R}(a M / q, m) \notin\left(\frac{s}{q} m, \frac{s+d+1}{q} m\right) .
$$

The condition $d>(q-10) / 10$ implies that

$$
\frac{s}{q}<\frac{s+d+1}{q}-\frac{1}{10}
$$

Let $\alpha$ belong to the interval

$$
\frac{s}{q} m<\alpha<\left(\frac{s+d+1}{q}-\frac{1}{10}\right) m .
$$

We claim that

$$
\mathrm{R}(a M / q-\alpha, m) \notin[0, m / 10) .
$$

Suppose otherwise; then we can write

$$
\frac{a}{q} M-\alpha=b m+r
$$

where $0 \leq r<m / 10$. Thus

$$
b m+\frac{s}{q} m<b m+\alpha \leq \frac{a}{q} M<b m+\alpha+\frac{m}{10}<b m+\frac{s+d+1}{q} m,
$$

as $\alpha$ satisfies (19). This contradicts (18), and establishes our claim. In fact we have shown that if $\alpha$ belongs to the interval (19), then $a M / q-\alpha \in \operatorname{Bad}(m)$.

Suppose now that $\alpha$ belongs to the interval $\pi \cdot L<\alpha<\Pi \cdot L$ (the second inequality in (16) ensures $\pi<\Pi$ ). To prove the proposition, all we have to show is that $\alpha$ satisfies the inequalities in (19) for all $m \in \mathcal{W}$. However, these follow straightforwardly from the fact that all $m \in \mathcal{W}$ belong to the interval (12).

A few remarks are in order concerning Proposition 5.2 and its proof:

- For simplicity we have only constructed the first interval in a ripple emanating from $a M / q$ in the negative direction. If inequalities (16) are satisfied with a significant margin, then it is possible to construct more intervals belonging to this ripple. Likewise, with a suitable modification of the assumptions one can also construct a ripple in the positive direction.

- The first inequality in (16) is imposed merely for simplicity; if it does not hold one can also construct ripples emanating from $a M / q$ after suitably modifying the second inequality in (16).

- The one indispensable assumption in Proposition 5.2 is the existence of a sequence

$$
\overline{s+1}, \overline{s+2}, \ldots, \overline{s+d}
$$


of consecutive residues belonging to $(\mathbb{Z} / q \mathbb{Z}) \backslash\{\overline{a M / m}: m \in \mathcal{W}\}$ of length $d$ that is roughly larger than $q / 10$. We shall show below that if there is no such sequence, then $\operatorname{Bad}(\mathcal{W})$ contains no elements in a neighborhood of $a M / q$.

Gaps. Let $a / q \in[0,1]$ be a rational in simplest form, and let

$$
\Phi_{a / q}: \mathcal{W} \rightarrow \mathbb{Z} / q \mathbb{Z}, \quad m \mapsto \overline{a(M / m)} .
$$

In view of the above, define the defect $d(\mathcal{W}, a / q)$ of $\mathcal{W}$ with respect to $a / q$ as the length of the longest sequence $\overline{s+1}, \overline{s+2}, \ldots, \overline{s+d}$ belonging to $(\mathbb{Z} / q \mathbb{Z}) \backslash \Phi_{a / q}(\mathcal{W})$. As $\mathcal{W} \neq \varnothing$, we have $d(\mathcal{W}, a / q)<q$. For example, if $\Phi_{a / q}$ is surjective then $d(\mathcal{W}, a / q)=0$, and if $\Phi_{a / q}(\mathcal{W})=(\mathbb{Z} / q \mathbb{Z})^{*}$ then $d(\mathcal{W}, a / q)=1$.

Lemma 5.3. With notation as above, let $d=d(\mathcal{W}, a / q)$. Let $x \in \mathbb{R}$. Then there is some element $m \in \mathcal{W}$ and an integer $k$ such that

$$
\left|x-\frac{a M}{q m}-k\right| \leq \frac{d+1}{2 q} .
$$

Proof. Let $u \in \mathbb{Z}$ satisfy $|u-q x| \leq \frac{1}{2}$. We first suppose that $d$ is even. Consider the sequence

$$
\overline{u-d / 2}, \overline{u-d / 2+1}, \overline{u-d / 2+2}, \ldots, \overline{u+d / 2}
$$

of $d+1$ elements of $\mathbb{Z} / q \mathbb{Z}$. By the definition of $d$, one of these equals $\Phi_{a / q}(m)$ for some $m \in \mathcal{W}$. Thus there is some integer $k$ such that

$$
\left|u-\frac{a M}{m}-k q\right| \leq \frac{d}{2} .
$$

As $|u-q x| \leq \frac{1}{2}$, the result follows.

Now suppose that $d$ is odd and $q x \geq u$ (the case $q x<u$ is similar). Consider the sequence

$$
\overline{u-(d-1) / 2}, \overline{u-(d-1) / 2+1}, \overline{u-(d-1) / 2+2}, \ldots, \overline{u+(d+1) / 2}
$$

which again has $d+1$ elements, and so there is some $m \in \mathcal{W}$ and some integer $k$ such that

$$
u-\frac{d-1}{2} \leq \frac{a M}{m}+k q \leq u+\frac{d+1}{2} .
$$

Since $0 \leq q x-u \leq \frac{1}{2}$, the lemma follows.

Lemma 5.4. Let

$$
m^{*}=\frac{38398}{13875} \cdot L,
$$

Then for all $m \in \mathcal{W}$,

$$
\left|\frac{L}{m}-\frac{L}{m^{*}}\right| \leq \frac{725}{38398} .
$$


Proof. By (12), the quantity $L / m$ belongs to the interval $\left[\frac{100}{292}, \frac{100}{263}\right]$. We have chosen $m^{*}$ so that $L / m^{*}$ is the midpoint of the interval. The lemma follows as $\frac{725}{38398}$ is half the length of the interval.

Proposition 5.5. With notation as above, let $d=d(\mathcal{W}, a / q)$ and suppose that $d<(q-10) / 10$. Let

$$
\mu=\frac{38398}{725}\left(\frac{1}{20}-\frac{d+1}{2 q}\right) .
$$

Then

$$
\left(\frac{a}{q} M-\mu L, \frac{a}{q} M+\mu L\right) \cap \operatorname{Bad}(\mathcal{W})=\varnothing .
$$

A few words are perhaps appropriate to help the reader appreciate the content of the proposition. We shall suppose that $q>11$. If $\# \mathcal{W}$ is large compared to $q$, then we expect that $\Phi_{a / q}$ is close to being surjective which forces $d$ to be small. If that is the case then $\mu$ should be close to $\frac{38398}{725 \times 20} \approx 2.64$. Suppose now that $\# \mathcal{W}$ is large, but that $q$ is much larger. Suppose also that the residues in the image $\Phi_{a / q}(\mathcal{W})$ are "randomly" distributed in $\mathbb{Z} / q \mathbb{Z}$. The quantity $d$ measures how large the gaps between these residues in the image can be, and we expect that $d$ should be around $q / \# \mathcal{W}$. We therefore expect that

$$
\mu \approx \frac{38398}{725}\left(\frac{1}{20}-\frac{1}{2 \cdot \# \mathcal{W}}\right)
$$

We see that $\mu$ should be positive if $\mathcal{W}$ has much more than 10 elements.

Proof of Proposition 5.5. The assumption $d<(q-10) / 10$ ensures that $\mu$ is positive. Let $y \in(a M / q-\mu L, a M / q+\mu L)$. We would to like to show that there is some $m \in \mathcal{W}$ such that $y \notin \operatorname{Bad}(m)$.

Write $y=a M / q+\beta$ where $|\beta|<\mu L$. Letting $x=\frac{1}{20}-\frac{\beta}{m^{*}}$ in Lemma 5.3, we deduce the existence of some integer $k$ and some element $m \in \mathcal{W}$ such that

$$
\left|\frac{\beta}{m^{*}}+\frac{a M}{q m}+k-\frac{1}{20}\right| \leq \frac{d+1}{2 q} .
$$

Thus

$$
\left|\frac{\beta}{m}+\frac{a M}{q m}+k-\frac{1}{20}\right| \leq \frac{d+1}{2 q}+\left|\frac{\beta}{m^{*}}-\frac{\beta}{m}\right| .
$$

Using $|\beta|<\mu L$, Lemma 5.4 and the definition of $\mu$ in (20), we see that

$$
\left|\frac{\beta}{m}+\frac{a M}{q m}+k-\frac{1}{20}\right|<\frac{1}{20} .
$$

Thus $y=a M / q+\beta$ belongs to the interval $-k m+(0, m / 10)$, showing that $y \notin \operatorname{Bad}(m)$ as required. 


\section{A first approach to computing $\operatorname{Bad}(\mathcal{W})$}

In this section $\mathcal{W}$ is a finite set of positive integers $m$. Associated to each $m \in \mathcal{W}$ are real numbers $0 \leq \varepsilon_{m}<\delta_{m}<1$. We shall write $\underset{\sim}{\varepsilon}=\left(\varepsilon_{m}\right)_{m \in \mathcal{W}}$ and $\underset{\sim}{\delta}=\left(\delta_{m}\right)_{m \in \mathcal{W}}$.

Lemma 6.1. Let $A<B$ be real numbers. For $m \in \mathcal{W}$, let

$$
q_{m}=\mathrm{Q}(A, m) \quad \text { and } \quad r_{m}=\mathrm{R}(A, m) .
$$

(a) Suppose $r_{n} \in\left[\varepsilon_{n} \cdot n, \delta_{n} \cdot n\right)$ for some $n \in \mathcal{W}$. Write $A^{\prime}=\min \left(\left(q_{n}+\delta_{n}\right) \cdot n, B\right)$. Then

$$
\operatorname{Bad}(\mathcal{W}) \cap[A, B)=\operatorname{Bad}(\mathcal{W}) \cap\left[A^{\prime}, B\right) .
$$

(b) Suppose $r_{m} \notin\left[\varepsilon_{m} \cdot m, \delta_{m} \cdot m\right)$ for all $m \in \mathcal{W}$. Define

$$
A_{m}=\left\{\begin{array}{ll}
\left(q_{m}+\varepsilon_{m}\right) \cdot m & \text { if } r_{m}<\varepsilon_{m} \cdot m, \\
\left(q_{m}+1+\varepsilon_{m}\right) \cdot m & \text { if } r_{m} \geq \delta_{m} \cdot m,
\end{array}\right\}, \quad A^{\prime}=\min \left(B, \min \left(A_{m}\right)_{m \in \mathcal{W}}\right) .
$$

Then

$$
\operatorname{Bad}(\mathcal{W}) \cap[A, B)=\left(\operatorname{Bad}(\mathcal{W}) \cap\left[A^{\prime}, B\right)\right) \cup\left[A, A^{\prime}\right) .
$$

Proof. Suppose $n \in \mathcal{W}$ satisfies $r_{n} \in\left[\varepsilon_{n} \cdot n, \delta_{n} \cdot n\right)$, and let $A^{\prime}$ be as in (a). By (5) we have

$$
\left(q_{n} \cdot n+\left[\varepsilon_{n} \cdot n, \delta_{n} \cdot n\right)\right) \cap \operatorname{Bad}(n)=\varnothing .
$$

Observe that $\left[A, A^{\prime}\right) \subseteq q_{n} \cdot n+\left[\varepsilon_{n} \cdot n, \delta_{n} \cdot n\right)$ and $\left[A, A^{\prime}\right) \subseteq[A, B)$. Part (a) follows.

Suppose now that $r_{m} \notin\left[\varepsilon_{m} \cdot m, \delta_{m} \cdot m\right)$ for all $m \in \mathcal{W}$, and let $A^{\prime}$ be as in (b). It is easy to check that $\mathrm{R}\left(A^{\prime \prime}, m\right) \notin\left[\varepsilon_{m} \cdot m, \delta_{m} \cdot m\right)$ for all $A^{\prime \prime} \in\left[A, A_{m}\right)$. From this we see that $\left[A, A^{\prime}\right) \subseteq \bigcap_{m \in \mathcal{W}} \operatorname{Bad}\left(m, \varepsilon_{m}, \delta_{m}\right)=\operatorname{Bad}(\mathcal{W})$. Part (b) follows.

Lemma 6.1 immediately leads us to the following algorithm.

Algorithm 1. To compute $\operatorname{Bad}(\mathcal{W}) \cap[A, B)$ as a disjoint union of intervals $\bigcup_{I \in \mathscr{I}} I$. Input: $A, B, \mathcal{W}, \underset{\sim}{\varepsilon}, \underset{\sim}{\delta}$.

Initialize $\mathscr{I} \leftarrow \varnothing$.

Repeat until $A=B$ :

(a) Loop through the elements $m \in \mathcal{W}$ computing $q_{m}=\mathrm{Q}(A, m), r_{m}=\mathrm{R}(A, m)$.

(b) If there is some $n \in \mathcal{W}$ such that $\varepsilon_{n} \cdot n \leq r_{n}<\delta_{n} \cdot n$ then for any such $n$ set

$$
A \leftarrow \min \left(\left(q_{n}+\delta_{n}\right) \cdot n, B\right)
$$

and go back to (a).

(c) Otherwise, let $A^{\prime}$ be as in (21). Set $\mathscr{I} \leftarrow \mathscr{I} \cup\left\{\left[A, A^{\prime}\right)\right\}$ and then $A \leftarrow A^{\prime}$. Go back to (a).

Output: $\mathscr{I}$. 
A heuristic analysis of Algorithm 1 and its running time. Let $x \in[0, M)$ and recall that $\mathrm{R}(x, m) \in[0, m)$. Moreover, $x \in \operatorname{Bad}(m)$ if and only if $\mathrm{R}(x, m) \in$ $[0, m) \backslash\left[\varepsilon_{m} \cdot m, \delta_{m} \cdot m\right)$. Thus the "probability" that $x$ belongs to $\operatorname{Bad}(m)$ is $1-\left(\delta_{m}-\varepsilon_{m}\right)$. Assuming "independence of events" we expect that the total length of intervals produced by Algorithm 1 is

$$
(B-A) \cdot \prod_{m \in \mathcal{W}}\left(1-\delta_{m}+\varepsilon_{m}\right) .
$$

To analyze the running time, we shall suppose parameter choices as in Lemma 2.3: namely $\varepsilon_{m}=0$ and $\delta_{m}=\frac{1}{10}$ for all $m \in \mathcal{W}$. Moreover, we shall suppose that the elements of $m \in \mathcal{W}$ belong to an interval (12) for some large positive $L$. By the above, the expected total length of the intervals produced by Algorithm 1 is $(B-A) \cdot 0.9^{\# \mathcal{W}}$. Moreover, we suppose that $\mathcal{W}$ is sufficiently large so that the length of the output should be negligible compared to $B-A$; this should mean that step (c) is relatively rare. We will estimate the expected number of times we loop through steps (a), (b). Note that in step (b), $A$ is increased by $0.1 \cdot n-r_{n}$. The remainder $r_{n}=\mathrm{R}(A, n)$ belongs to $[0,0.1 \cdot n)$. We regard the increase as a product $\left(0.1-r_{n} / n\right) \cdot n$. Treating $r_{n} / n$ as a random variable uniformly distributed in $[0,0.1)$ and $n$ as a random variable uniformly distributed in interval (12), we see that the expected increase is $0.05 \cdot(2.63+2.92) L / 2=0.13875 \cdot L$. A standard probability theory argument that we omit tells us that the expected number of times the algorithm loops through steps (a), (b) is roughly

$$
\frac{B-A}{0.13875 L} \approx \frac{7(B-A)}{L} .
$$

We now suppose that $K$ is very large, and we would like to compute the intersection $\operatorname{Bad}(\mathcal{W}) \cap[0, M)$ for some set $\mathcal{W}$ where we hope that the hypotheses of Proposition 2.2 and Lemma 2.3 are satisfied. In particular, we take $L=K^{\frac{1}{3}}$. The number of steps should be around $7 M / K^{\frac{1}{3}}$. We have to choose $\mathcal{W}$ so that $M=$ $\operatorname{lcm}(\mathcal{W})$ is much larger than $K$ (see (7) and just above it). Thus the number of steps to compute $\operatorname{Bad}(\mathcal{W})$ is much greater than $K^{\frac{2}{3}}$. For $K=\exp (524)$, the expected number of steps is larger than $10^{150}$, which makes the computation entirely impractical.

\section{A refined approach to computing $\operatorname{Bad}(\mathcal{W})$ : The tower}

In this section we let $\mathcal{W}$ be a set of positive integers with $M=\operatorname{lcm}(\mathcal{W})$. Let $M_{0}, M_{1}, M_{2}, \ldots, M_{r}$ be positive integers such that $M_{i} \mid M_{i+1}$ and $M_{r}=M$. Write $p_{i}=M_{i+1} / M_{i}$. In our later computations the $p_{i}$ will be primes, but we need not assume that yet. Let

$$
\mathcal{W}_{i}=\left\{m \in \mathcal{W}: m \mid M_{i}\right\}
$$


We suppose that $M_{i}=\operatorname{lcm}\left(\mathcal{W}_{i}\right)$. Write $\mathcal{U}_{i}=\mathcal{W}_{i+1} \backslash \mathcal{W}_{i}$. Recall (Lemmas 4.3 and 4.4) that we have natural surjections $\pi_{M_{j}, M_{i}}:\left[0, M_{j}\right) \rightarrow\left[0, M_{i}\right)$ whenever $j \geq i$, and that these restrict to give maps (not necessarily surjections) $\operatorname{Bad}\left(\mathcal{W}_{j}\right) \rightarrow \operatorname{Bad}\left(\mathcal{W}_{i}\right)$. For ease of notation we shall denote $\pi_{M_{j}, M_{i}}$ simply by $\pi_{j, i}$. We shall refer to the sequence of inclusions (10) as a tower leading up to $\operatorname{Bad}(\mathcal{W})$, and use this to compute $\operatorname{Bad}(\mathcal{W})$.

Lemma 7.1. Let $0 \leq i \leq r-1$. Suppose $\mathscr{I}_{i}$ is a finite set of disjoint subintervals of $\left[0, M_{i}\right)$ such that

$$
\operatorname{Bad}\left(\mathcal{W}_{i}\right) \cap\left[0, M_{i}\right)=\bigcup_{I \in \mathscr{I}_{i}} I
$$

Then

$$
\operatorname{Bad}\left(\mathcal{W}_{i+1}\right) \cap\left[0, M_{i+1}\right)=\bigcup_{I \in \mathscr{I}_{i}} \bigcup_{k=0}^{p_{i}-1}\left(\left(k \cdot M_{i}+I\right) \cap \operatorname{Bad}\left(\mathcal{U}_{i}\right)\right) .
$$

Proof. This is immediate from Lemmas 4.3 and 4.4 .

Lemma 7.1 immediately leads us to the following algorithm.

Algorithm 2. The following computes a finite set $\mathscr{I}=\mathscr{I}_{r}$ of subintervals of $[0, M)$ such that $\operatorname{Bad}(\mathcal{W}) \cap[0, M)=\bigcup_{I \in \mathscr{I}} I$.

Input: $\mathcal{W}_{0}, \ldots, \mathcal{W}_{r}=\mathcal{W}, \underset{\sim}{\varepsilon}, \underset{\sim}{\delta}$.

Initialize: $\mathscr{I}_{0}$ to be the set of disjoint intervals whose union is $\operatorname{Bad}\left(\mathcal{W}_{0}\right) \cap\left[0, M_{0}\right)$, which is computed using Algorithm 1.

Initialize: $i \leftarrow 0$.

Repeat until $i=r$ :

(a) $\mathscr{I}_{i+1} \leftarrow \varnothing$.

(b) for $I \in \mathscr{I}_{i}$ and $k \in\left\{0, \ldots, p_{i}-1\right\}$, compute, using Algorithm 1, a finite set $\mathscr{I}^{\prime}$ of subintervals of $\left[0, M_{i+1}\right)$ such that $\left(k \cdot M_{i}+I\right) \cap \operatorname{Bad}\left(\mathcal{U}_{i}\right)=\bigcup_{I^{\prime} \in \mathscr{I}^{\prime}} I^{\prime}$; let $\mathscr{I}_{i+1} \leftarrow \mathscr{I}_{i+1} \cup \mathscr{I}^{\prime}$.

(c) $i \leftarrow i+1$.

Output: $\mathscr{I}=\mathscr{I}_{r}$.

A heuristic analysis of Algorithm 2 and its running time. We shall suppose, as in Lemma 2.3, that $\varepsilon_{m}=0$ and $\delta_{m}=\frac{1}{10}$ for all $m \in \mathcal{W}$. Write $n_{i}=\# \mathcal{W}$. We assume that the elements of $\mathcal{W}_{i}, \mathcal{U}_{i}$ belong to an interval of the form $\left[\frac{263 L}{100}, \frac{292 L}{100}\right]$ for some large $L$. By our previous analysis, we expect that we can compute $\mathscr{I}_{0}$ in roughly $7 M_{0} / L$ steps. The total length $\ell\left(\mathscr{I}_{0}\right)$ of the intervals in $\mathscr{I}_{0}$ should roughly be $0.9^{n_{0}} M_{0}$. In Step (b) of the algorithm, we will replace each $I \in \mathscr{I}_{0}$ with $p_{0}$ intervals of the same length, and then apply Algorithm 1 to each. Thus we expect that the number of steps to compute $\mathscr{I}_{1}$ to be roughly 


$$
\frac{7 p_{0} \cdot 0.9^{n_{0}} \cdot M_{0}}{L} \approx \frac{7 M_{1} \cdot 0.9^{n_{0}}}{L} .
$$

The total length of the intervals in $\mathscr{I}_{1}$ should be roughly $M_{1} \cdot 0.9^{n_{1}}$. It is now apparent that the total number of steps should be around

$$
(7 / L) \cdot\left(M_{0}+M_{1} \cdot 0.9^{n_{0}}+M_{2} \cdot 0.9^{n_{1}}+\cdots+M_{r} \cdot 0.9^{n_{r-1}}\right) .
$$

\section{A large computation}

Let $M^{*}$ be the product of all primes $p \leq 167$ that are $\equiv 5(\bmod 6)$, and $\mathcal{W}^{*}$ be as in (11). In this section we compute $\operatorname{Bad}\left(\mathcal{W}^{*}\right) \cap\left[0, M^{*}\right)$, using a tower and Algorithm 2. As explained in Section 3, the result of this computation will be reused again and again in Section 9. Let

$$
M_{0}=5 \times 11 \times 17 \times 23 \times 29 \times 41 \times 47 \times 53 \times 59 \times 71 \times 83 \times 89,
$$

which is the product of the primes $<100$ that are $\equiv 5(\bmod 6)$. Let

$$
\begin{gathered}
M_{1}=101 \cdot M_{0}, \quad M_{2}=107 \cdot M_{1}, \quad M_{3}=113 \cdot M_{2}, \quad M_{4}=131 \cdot M_{3}, \\
M_{5}=137 \cdot M_{4}, \quad M_{6}=149 \cdot M_{5}, \quad M^{*}=M_{7}=167 \cdot M_{6} .
\end{gathered}
$$

We let

$$
\mathcal{W}_{i}=\left\{m \mid M_{i}: 265 \times 10^{9} \leq m \leq 290 \times 10^{9}\right\} .
$$

Thus $\mathcal{W}_{0} \subseteq \cdots \subseteq \mathcal{W}_{7}=\mathcal{W}^{*}$. We checked that $M_{i}=\operatorname{lcm}\left(\mathcal{W}_{i}\right)$. Table 1 gives the cardinalities of the $\mathcal{W}_{i}$. We use this tower and Algorithm 2 to compute $\operatorname{Bad}\left(\mathcal{W}^{*}\right) \cap\left[0, M^{*}\right)$. By our heuristic in the previous section, the number of steps needed for this computation should very roughly be equal to $6.0 \times 10^{10}$, which is the sum of the entries of the table's third column. It appears from this estimate that the computation can be done in reasonable time.

We wrote simple implementations of Algorithms 1 and 2 for the computer algebra system Magma [Bosma et al. 1997]. We divided the interval [0, $M_{0}$ ) into 59000 subintervals of equal length and ran our program on each of these intervals $\left[A_{k-1}, A_{k}\right)$ successively computing $\operatorname{Bad}\left(\mathcal{W}_{i}\right) \cap \pi_{i, 0}^{-1}\left(\left[A_{k-1}, A_{k}\right)\right)$ for $i=0, \ldots, 7$. Our computation was distributed over 59 processors (on a 64 core machine with 2500MHz AMD Opteron Processors). Note that

$$
\operatorname{Bad}\left(\mathcal{W}_{i}\right) \cap\left[0, M_{i}\right)=\bigcup_{k=1}^{59000} \operatorname{Bad}\left(\mathcal{W}_{i}\right) \cap \pi_{i, 0}^{-1}\left(\left[A_{k-1}, A_{k}\right)\right) ;
$$

thus our computation gives us a decomposition of $\operatorname{Bad}\left(\mathcal{W}_{i}\right) \cap\left[0, M_{i}\right)$ as a union of disjoint intervals. The total CPU time for the computation was around 18,300 hours, but as we distributed it over 59 processors, it was over in less than two weeks. 


\begin{tabular}{cccc}
\hline$i$ & $\log _{10}\left(M_{i}\right)$ & $n_{i}=\# \mathcal{W}_{i}$ & $7 M_{i} 0.9^{n_{i-1}} / 10^{11}$ \\
\hline 0 & 18.3 & 16 & $1.4 \times 10^{9}$ \\
1 & 20.3 & 38 & $2.6 \times 10^{9}$ \\
2 & 22.3 & 83 & $2.7 \times 10^{10}$ \\
3 & 24.4 & 149 & $2.7 \times 10^{10}$ \\
4 & 26.5 & 250 & $3.4 \times 10^{9}$ \\
5 & 28.6 & 401 & $1.1 \times 10^{7}$ \\
6 & 30.8 & 620 & $2.0 \times 10^{2}$ \\
7 & 33.0 & 911 & $3.2 \times 10^{-6}$ \\
\hline
\end{tabular}

Table 1. The $M_{i}$ and the $\mathcal{W}_{i}$ are given at the beginning of Section 8 . The third column gives an estimate for the number of steps needed to compute $\operatorname{Bad}\left(\mathcal{W}_{i}\right) \cap\left[0, M_{i}\right)$ from $\operatorname{Bad}\left(\mathcal{W}_{i-1}\right) \cap\left[0, M_{i-1}\right)$ according to the heuristic analysis at the end of Section 7.

Lemma 8.1. There are sequences $\left(B_{j}\right)_{j=1}^{854}$ and $\left(C_{j}\right)_{j=1}^{854}$ contained in $\left[0, M^{*}\right]$ such that

$$
B_{1}<C_{1}<B_{2}<C_{2}<\cdots<B_{854}<C_{854}
$$

and

$$
\operatorname{Bad}\left(\mathcal{W}^{*}\right) \cap\left[0, M^{*}\right)=\bigcup_{j=1}^{854}\left[B_{j}, C_{j}\right),
$$

with total length $\sum_{j=1}^{854}\left(C_{j}-B_{j}\right)=20382195221000 \frac{6}{10}$.

Proof. As indicated by Table 2, our computation gives $\operatorname{Bad}\left(\mathcal{W}^{*}\right) \cap\left[0, M^{*}\right)$ as a union of 861 intervals disjoint subintervals of $\left[0, M^{*}\right)$. Among these there are 7 pairs of the form $[\alpha, \beta) \cup[\beta, \gamma)$, where the values of $\beta$ are of the form $\beta^{\prime} \cdot M^{*} / 59000$ with

$$
\beta^{\prime}=7375,14750,22125,29500,36875,44250,51625 .
$$

These subdivisions are clearly a result of our original subdivision of interval $\left[0, M_{0}^{*}\right)$ into 59000 subintervals of equal length. We simply replace the pairs $[\alpha, \beta) \cup[\beta, \gamma)$ with $[\alpha, \gamma)$ so that $\operatorname{Bad}\left(\mathcal{W}^{*}\right) \cap\left[0, M^{*}\right)$ is expressed as a union of 854 intervals. This simplification of course preserves the total length of intervals.

Remarks and sanity checks. Our computations are done with exact arithmetic. The reader will note by looking back at Algorithms 1 and 2 (and recalling that all $\varepsilon_{m}=0$ and $\delta_{m}=m / 10$ ) that the end points of the intervals encountered will be rationals with denominators that are divisors of 10, except for the $A_{k}$ appearing in our original subdivision which have denominators that are divisors of 59000. As a 


\begin{tabular}{rrrcc}
\hline$i$ & \multicolumn{1}{c}{$\# \mathscr{I}_{i}$} & $\ell_{i}=\ell\left(\operatorname{Bad}\left(\mathcal{W}_{i}\right) \cap\left[0, M_{i}\right)\right)$ & $\ell_{i} / M_{i}$ & $0.9^{n_{i}}$ \\
\hline 0 & 23458002 & $365300497739376385 \frac{8}{10}$ & $1.85 \times 10^{-1}$ & $1.85 \times 10^{-1}$ \\
1 & 553209618 & $3625384986862035664 \frac{4}{10}$ & $1.82 \times 10^{-2}$ & $1.82 \times 10^{-2}$ \\
2 & 1106375245 & $3313998145602553709 \frac{1}{10}$ & $1.56 \times 10^{-4}$ & $1.59 \times 10^{-4}$ \\
3 & 209982392 & $350826426611537217 \frac{1}{10}$ & $1.46 \times 10^{-7}$ & $1.52 \times 10^{-7}$ \\
4 & 1062201 & $1076402154947217 \frac{8}{10}$ & $3.41 \times 10^{-12}$ & $3.64 \times 10^{-12}$ \\
5 & 904 & $20663973893432 \frac{1}{10}$ & $4.78 \times 10^{-16}$ & $4.48 \times 10^{-19}$ \\
6 & 870 & $20504346087851 \frac{7}{10}$ & $3.19 \times 10^{-18}$ & $4.27 \times 10^{-29}$ \\
7 & 861 & $20382195221000 \frac{6}{10}$ & $1.90 \times 10^{-20}$ & $2.07 \times 10^{-42}$ \\
\hline
\end{tabular}

Table 2. Some details for the computation described Section 8. The second column gives \# $\mathscr{I}_{i}$, where $\mathscr{I}_{i}$ is a disjoint collection of intervals $\bigcup \mathscr{I}_{i}=\operatorname{Bad}\left(\mathcal{W}_{i}\right) \cap\left[0, M_{i}\right)$. The third column gives the total length $\ell_{i}$ of these intervals. The fourth column gives the ratio $\ell_{i} / M_{i}$. According to the heuristic at the end of Section 6, this ratio should approximately equal $0.9^{n_{i}}$ which is given in the last column (here $n_{i}=\# \mathcal{W}_{i}$ as in Table 1). We explain the discrepancy between the last two columns in the remarks on page 2111 .

check on our computations, we verify that our results for $\operatorname{Bad}\left(\mathcal{W}^{*}\right) \cap\left[0, M^{*}\right)$ are consistent with Proposition 5.1. The set $\mathcal{W}^{*}$ satisfies

$$
\min \left(\mathcal{W}^{*}\right)=265024970473 \text { and } \max \left(\mathcal{W}^{*}\right)=289916573827
$$

We take $L=\min \left(\mathcal{W}^{*}\right) \cdot \frac{100}{263}$. It turns out that $L>\max \left(\mathcal{W}^{*}\right) \cdot \frac{100}{292}$. Thus $\mathcal{W}^{*}$ is contained in the interval (12) for this value of $L$. Proposition 5.1 yields a total of 103 intervals of the form $\left(a M^{*} / q+\psi_{k} \cdot L, a M^{*} / q+\Psi_{k} \cdot L\right)$ that must be contained in $\operatorname{Bad}\left(\mathcal{W}^{*}\right) \cap\left[0, M^{*}\right)$. We checked that each of these is contained in one of the 854 intervals produced by our computation. It is instructive to compare the fourth and fifth columns of Table 2. According to our heuristic, the total length $\ell\left(\operatorname{Bad}\left(\mathcal{W}_{i}\right) \cap\left[0, M_{i}\right)\right)$ should be around $M_{i} \cdot 0.9^{n_{i}}$ (with $n_{i}=\# \mathcal{W}_{i}$ ) and therefore we expect the two columns to be roughly the same. From the table, we see that this heuristic is remarkably accurate for $0 \leq i \leq 4$, and extremely inaccurate for $i \geq 5$. An explanation for this is provided by the ripples. The total length of the intervals contained in $\operatorname{Bad}\left(\mathcal{W}_{i}\right) \cap\left[0, M_{i}\right)$ produced by Proposition 5.1 is $\approx 24.9 L$. Now

$$
\frac{24.9 L}{M_{5}}=5.8 \times 10^{-17}, \quad \frac{24.9 L}{M_{6}}=4.0 \times 10^{-19}, \quad \frac{24.9 L}{M_{7}}=2.3 \times 10^{-21},
$$


which does provide an explanation for the discrepancy between the two columns. Proposition 5.2 (with $\mathcal{W}^{*}$ and $M^{*}$ in place of $\mathcal{W}$ and $M$ ) produces 172 intervals with $11 \leq q \leq 100$ with total length $\approx 17.8 \mathrm{~L}$. We checked that each of these is also contained in one of the 854 intervals produced by our computation.

According to the overall philosophy of Section 5, the set $\operatorname{Bad}\left(\mathcal{W}^{*}\right) \cap\left[0, M^{*}\right)$ should be concentrated in short intervals around rational multiples $(a / q) \cdot M^{*}$ with $q$ small. To test this, we computed, using continued fractions, the best rational approximation to $\left(B_{i}+C_{i}\right) /\left(2 M^{*}\right)$ with denominator at most $10^{20}$, for $1 \leq i \leq 854$. The largest denominator we found was 42 .

The reader is probably wondering, given that we are employing 59 processors, why we have subdivided $\left[0, M_{0}\right)$ into 59,000 intervals instead of 59 intervals. This was done purely for memory management reasons. A glance at Table 2 will show the reader that there is an explosion of intervals at levels $i=1,2,3$. By dividing $\left[0, M_{0}\right)$ into 59,000 subintervals, we only need to store roughly $\frac{1}{59000}$-th of the intervals appearing at levels $i$ at any one time per processor, and so only need to store around $\frac{1}{1000}$-th of these intervals in the memory at any one time.

\section{Proof of Theorem 1 for $N \geq\left(\frac{9}{10}\right)^{3998} \cdot \exp (524) \approx 4.28 \times 10^{44}$}

The reader might at this point find it helpful to review the first paragraph of Section 2 as well as the plan in Section 3. Let $\mathscr{K}=\exp (524)$. In this section we prove Theorem 1 for $N \geq\left(\frac{9}{10}\right)^{3998} \mathscr{K}$. We shall divide the interval $\left(\frac{9}{10}\right)^{3998} \mathscr{K} \leq N \leq \mathscr{K}$ into subintervals $\left(\frac{9}{10}\right)^{n+1} \mathscr{K} \leq N \leq\left(\frac{9}{10}\right)^{n} \mathscr{K}$ with $0 \leq n \leq 3997$. We apply Proposition 2.2 and Lemma 2.3 to prove that all odd integers in the interval $\left(\frac{9}{10}\right)^{n+1} \mathscr{K} \leq N \leq\left(\frac{9}{10}\right)^{n} \mathscr{K}$ are sums of seven nonnegative cubes.

Lemma 9.1. Let $0 \leq n \leq 3997$. Let $K=\left(\frac{9}{10}\right)^{n} \cdot \mathscr{K}$. There exists an integer $\kappa$ that satisfies:

(a) $\kappa$ is squarefree.

(b) $3 \mid \kappa$.

(c) $\kappa / 3$ is divisible only by primes $q \equiv 5(\bmod 6)$ that satisfy $q>167$.

(d) $\kappa$ belongs to the interval

$$
\frac{263}{265} \cdot \frac{K^{\frac{1}{3}}}{10^{11}} \leq \kappa \leq \frac{292}{290} \cdot \frac{K^{\frac{1}{3}}}{10^{11}} .
$$

Proof. We proved the lemma using a Magma script. Let $I_{1}, I_{2}$ be the lower and upper bounds for $\kappa$ in (23). If $I_{2}<10^{7}$ then our script uses brute enumeration of integers in the interval $\left[I_{1}, I_{2}\right]$ to find a suitable $\kappa$. Otherwise, the script takes $\tau$ to be a product of consecutive primes $\equiv 5(\bmod 6)$ starting with 173 up to a certain bound, 
and keeps increasing the bound until $I_{2} / \tau<10^{7}$. It then loops through the integers $I_{\frac{1}{3}} \tau \leq \mu \leq I_{\frac{2}{3}} \tau$ until it finds one such that $\kappa=3 \mu \tau$ satisfies conditions (a), (b), (c).

Remark. For $n=3998$, the interval in (23) is $7481.6 \ldots \leq \kappa \leq 7590.5 \ldots$, which is too short for the existence of a suitable $\kappa$. This is also the case for most values of $n$ that are $\geq 3998$.

Lemma 9.2. Let $0 \leq n \leq 3997$ and let $\kappa$ be as in Lemma 9.1. Let $\mathcal{W}^{*}$ and $M^{*}$ be as in Lemma 8.1. Let

$$
\mathcal{W}_{0}=\left\{\kappa \cdot m^{*}: m^{*} \in \mathcal{W}^{*}\right\} \quad \text { and } \quad M_{0}=\operatorname{lcm}\left(\mathcal{W}_{0}\right)=\kappa M^{*} .
$$

Let $\varepsilon_{m}=0$ and $\delta_{m}=\frac{1}{10}$ for all $m \in \mathcal{W}_{0}$. Then $m \in \mathcal{W}_{0}$ satisfy the conditions ( $\left.i\right)-(v i)$ of Section 2, where

$$
K_{1}=\left(\frac{9}{10}\right)^{n+1} \cdot \mathscr{K} \quad \text { and } \quad K_{2}=\left(\frac{9}{10}\right)^{n} \cdot \mathscr{K} .
$$

Moreover,

$$
\operatorname{Bad}\left(\mathcal{W}_{0}\right) \cap\left[0, M_{0}\right)=\bigcup_{j=1}^{854}\left[\kappa \cdot B_{j}, \kappa \cdot C_{j}\right),
$$

where the $B_{j}$ and $C_{j}$ are as in Lemma 8.1.

Proof. All $m^{*} \in \mathcal{W}^{*}$ are squarefree and divisible only by primes $q \leq 167$ satisfying $q \equiv 5(\bmod 6)$. Thus conditions (i)-(iii) of Section 2 are satisfied by $m \in \mathcal{W}_{0}$. As we are taking $\varepsilon_{m}=0$ and $\delta_{m}=\frac{1}{10}$, to verify conditions (iv)-(vi) we may apply Lemma 2.3. For this we need only check that (9) holds for $m \in \mathcal{W}_{0}$, where $K=K_{2}$. This immediately follows from (23) and the fact that $\mathcal{W}^{*} \subset\left[265 \times 10^{9}, 290 \times 10^{9}\right]$.

Finally, by Lemma 4.2,

$$
\operatorname{Bad}\left(\mathcal{W}_{0}\right) \cap\left[0, M_{0}\right)=\kappa \cdot\left(\operatorname{Bad}\left(\mathcal{W}^{*}\right) \cap\left[0, M^{*}\right)\right) .
$$

Lemma 8.1 completes the proof.

Our Magma script for proving Theorem 1 in the range $K_{1} \leq N \leq K_{2}$ proceeds as follows. We inductively construct a tower $\mathcal{W}_{0} \subset \mathcal{W}_{1} \subset \mathcal{W}_{2} \subset \ldots$. Observe that

$$
\frac{\ell\left(\operatorname{Bad}\left(\mathcal{W}_{0}\right) \cap\left[0, M_{0}\right)\right)}{M_{0}}=\frac{\ell\left(\operatorname{Bad}\left(\mathcal{W}^{*}\right) \cap\left[0, M^{*}\right)\right)}{M^{*}} \approx 1.90 \times 10^{-20},
$$

thus the computation of the previous section has already substantially depleted the interval $\left[0, M_{0}\right)$. Given $\mathcal{W}_{i}$, and $M_{i}$, we let $p_{i}$ be the smallest prime $\equiv 5$ (mod 6) that does not divide $M_{i}$ and let $M_{i+1}=p_{i} M_{i}$. The script then writes down positive integers $m$ belonging to the interval (9), such that $m \mid M_{i+1}$ and $3 p_{i} \mid m$. It is not necessary or practical to find all such integers, but we content ourselves with finding around $3 \log \left(p_{i}\right) / \log \left(0.9^{-1}\right)$ of them; we explain this choice shortly. These $m$ will form the set $\mathcal{U}_{i}$ and we take $\mathcal{W}_{i+1}=\mathcal{W}_{i} \cup \mathcal{U}_{i}$. The script then 
applies our implementation of Algorithm 2 to compute $\operatorname{Bad}\left(\mathcal{W}_{i+1}\right) \cap\left[0, M_{i+1}\right)$ as a union of disjoint intervals. Our heuristic analysis of Algorithm 2 suggests that $\ell\left(\operatorname{Bad}\left(\mathcal{W}_{i+1}\right) \cap\left[0, M_{i+1}\right)\right)$ should roughly equal $p_{i} \cdot 0.9^{\# \mathcal{U}_{i}} \cdot \ell\left(\operatorname{Bad}\left(\mathcal{W}_{i}\right) \cap\left[0, M_{i}\right)\right)$. We desire the total length of the intervals to decrease in each step of the tower, so we should require $\# \mathcal{U}_{i}>\log \left(p_{i}\right) / \log \left(0.9^{-1}\right)$. Experimentation suggests that requiring $\# \mathcal{U}_{i} \approx 3 \log \left(p_{i}\right) / \log \left(0.9^{-1}\right)$ provides good control of both the total length of $\operatorname{Bad}\left(\mathcal{W}_{i}\right) \cap\left[0, M_{i}\right)$ and the number of intervals that make it up. Our script continues to build the tower and compute successive $\operatorname{Bad}\left(\mathcal{W}_{i}\right) \cap\left[0, M_{i}\right)$ until it finds $\mathcal{W}=\mathcal{W}_{i}$ and $M=M_{i}$ that satisfy (7) for some set of rationals $\mathfrak{S} \subset[0,1]$ with denominators bounded by $\sqrt[3]{M / 2 K}$. Specifically, once $M_{i}>2 K$, for each of the disjoint intervals $[\alpha, \beta)$ that make up $\operatorname{Bad}(\mathcal{W}) \cap[0, M)$, the script uses continued fractions to compute the best rational approximation $a / q$ to $(\alpha+\beta) / 2 M$ with $q \leq \sqrt[3]{M / 2 K}$, and then checks whether

$$
[\alpha, \beta) \subseteq(a M / q-\sqrt[3]{M / 16} / q, a M / q+\sqrt[3]{M / 16} / q) .
$$

The script continues constructing the tower until this criterion is satisfied for all the intervals making up $\operatorname{Bad}(\mathcal{W})$. It then follows from Proposition 2.2 that all odd integers in the range $\mathscr{K} \cdot\left(\frac{9}{10}\right)^{n+1} \leq N \leq \mathscr{K} \cdot\left(\frac{9}{10}\right)^{n}$ are sums of seven nonnegative cubes. We again distributed the computation among 59 processors on the aforementioned machine, with each processor handling an appropriate portion of the range $0 \leq n \leq 3997$. The script succeeded in finding an appropriate $\mathcal{W}$ for all $n$ in this range. The entire CPU time was around 10,000 hours, but as the computation was distributed among 59 processors the actual time was around 7 days.

We give more details for the case $n=0$. Thus $K=\mathscr{K}=\exp (524)$, and we would like to show, using Proposition 2.2 that all odd integers $9 K / 10 \leq N \leq K$ are sums of seven nonnegative cubes. The routine described in the proof of Lemma 9.1 gives the following suitable value for $\kappa$ :

$\kappa=3 \times 173 \times 179 \times 191 \times 197 \times 227 \times 233 \times 239 \times 251 \times 257 \times 263 \times 269 \times 281 \times 293$

$\times 311 \times 317 \times 347 \times 353 \times 359 \times 383 \times 389 \times 401 \times 419 \times 431 \times 443 \times 207443$.

Table 3 gives some of the details for the computation. We take $\mathcal{W}=\mathcal{W}_{48}$. Then $\# \mathcal{W}=\# \mathcal{W}_{0}+\sum \# \mathcal{U}_{i}=9943$, and

$\ell(\operatorname{Bad}(\mathcal{W}) \cap[0, M))$

$=1245937137395549638824015714140403151401411370898968055175937887691670913319978 \frac{1}{2}$

$\approx 1.25 \times 10^{78}$.

In comparison,

$$
M=M_{48} \approx 1.64 \times 10^{235} \text { and } K=3.72 \times 10^{227} .
$$




\begin{tabular}{|rcccc|ccccc|}
\hline$i$ & $p_{i-1}$ & $\# \mathcal{U}_{i-1}$ & $N$ & $\ell_{i} / M_{i}$ & $i$ & $p_{i-1}$ & $\# \mathcal{U}_{i-1}$ & $N$ & $\ell_{i} / M_{i}$ \\
\hline 0 & - & - & 854 & $1.90 \times 10^{-20}$ & 25 & 761 & 189 & 729 & $9.93 \times 10^{-90}$ \\
1 & 449 & 174 & 775 & $3.73 \times 10^{-23}$ & 26 & 773 & 190 & 729 & $1.28 \times 10^{-92}$ \\
2 & 461 & 175 & 745 & $7.94 \times 10^{-26}$ & 27 & 797 & 191 & 729 & $1.61 \times 10^{-95}$ \\
3 & 467 & 176 & 740 & $1.70 \times 10^{-28}$ & 28 & 809 & 191 & 729 & $1.99 \times 10^{-98}$ \\
4 & 479 & 176 & 735 & $3.54 \times 10^{-31}$ & 29 & 821 & 192 & 729 & $2.43 \times 10^{-101}$ \\
5 & 491 & 177 & 732 & $7.20 \times 10^{-34}$ & 30 & 827 & 192 & 729 & $2.93 \times 10^{-104}$ \\
6 & 503 & 178 & 730 & $1.42 \times 10^{-36}$ & 31 & 839 & 192 & 729 & $3.50 \times 10^{-107}$ \\
7 & 509 & 178 & 730 & $2.80 \times 10^{-39}$ & 32 & 857 & 193 & 729 & $4.08 \times 10^{-110}$ \\
8 & 521 & 179 & 730 & $5.38 \times 10^{-42}$ & 33 & 863 & 193 & 729 & $4.73 \times 10^{-113}$ \\
9 & 557 & 181 & 730 & $9.65 \times 10^{-45}$ & 34 & 881 & 194 & 729 & $5.36 \times 10^{-116}$ \\
10 & 563 & 181 & 731 & $1.71 \times 10^{-47}$ & 35 & 887 & 194 & 729 & $6.05 \times 10^{-119}$ \\
11 & 569 & 181 & 730 & $3.01 \times 10^{-50}$ & 36 & 911 & 195 & 729 & $6.64 \times 10^{-122}$ \\
12 & 587 & 182 & 729 & $5.13 \times 10^{-53}$ & 37 & 929 & 195 & 729 & $7.14 \times 10^{-125}$ \\
13 & 593 & 182 & 729 & $8.64 \times 10^{-56}$ & 38 & 941 & 195 & 729 & $7.59 \times 10^{-128}$ \\
14 & 599 & 183 & 729 & $1.44 \times 10^{-58}$ & 39 & 947 & 196 & 729 & $8.02 \times 10^{-131}$ \\
15 & 617 & 183 & 729 & $2.34 \times 10^{-61}$ & 40 & 953 & 196 & 729 & $8.41 \times 10^{-134}$ \\
16 & 641 & 185 & 729 & $3.64 \times 10^{-64}$ & 41 & 971 & 196 & 729 & $8.66 \times 10^{-137}$ \\
17 & 647 & 185 & 729 & $5.63 \times 10^{-67}$ & 42 & 977 & 197 & 729 & $8.87 \times 10^{-140}$ \\
18 & 653 & 185 & 729 & $8.62 \times 10^{-70}$ & 43 & 983 & 197 & 729 & $9.02 \times 10^{-143}$ \\
19 & 659 & 185 & 729 & $1.31 \times 10^{-72}$ & 44 & 1013 & 198 & 729 & $8.91 \times 10^{-146}$ \\
20 & 677 & 186 & 729 & $1.93 \times 10^{-75}$ & 45 & 1019 & 198 & 729 & $8.74 \times 10^{-149}$ \\
21 & 683 & 186 & 729 & $2.83 \times 10^{-78}$ & 46 & 1031 & 198 & 729 & $8.48 \times 10^{-152}$ \\
22 & 701 & 187 & 729 & $4.04 \times 10^{-81}$ & 47 & 1049 & 199 & 729 & $8.08 \times 10^{-155}$ \\
23 & 719 & 188 & 729 & $5.61 \times 10^{-84}$ & 48 & 1061 & 199 & 729 & $7.62 \times 10^{-158}$ \\
24 & 743 & 189 & 729 & $7.55 \times 10^{-87}$ & & & & & \\
\hline
\end{tabular}

Table 3. details for the computation for the case $n=0$. For each $i \geq 1$, our script computes $\operatorname{Bad}\left(\mathcal{W}_{i}\right)$ as a disjoint union of subintervals of $\left[0, M_{i}\right)$. The number of intervals, $N$, is given in the fourth column. The fifth column gives, to 3 significant figures, the ratio $\ell_{i} / M_{i}$ where $\ell_{i}=\ell\left(\operatorname{Bad}\left(\mathcal{W}_{i}\right) \cap\left[0, M_{i}\right)\right)$.

The set $\mathfrak{S}$ as in (7) turns out be precisely the set of 171 rationals $a / q \in[0,1]$ with denominators $q$ belonging to

$$
1,2,3,4,5,6,7,8,9,10,12,13,14,15,16,18,19,21,24,26,28,30,36,42 .
$$

As a check on our results, we apply Proposition 5.2 to show that there is an interval close to $(a / 42) \cdot M$ for $1 \leq a \leq 41$ with $\operatorname{gcd}(a, 42)=1$. Our $\mathcal{W}$ and $M$ satisfy the hypotheses of Section 5 with $L=K^{\frac{1}{3}}$. Note that $3|m| M$ for all $m \in \mathcal{W}$. As $M$ is squarefree, we have $3 \nmid(M / m)$. Moreover, all the prime divisors of $M / 3$ 
are $\equiv 5(\bmod 6)$. It follows that $\operatorname{gcd}(a M / m, 42)=1$ for all $m \in \mathcal{W}$. Let $q=42$ and $s=d=5$ in Proposition 5.2; hypothesis (16) is trivially satisfied. Now $s+1, \ldots, s+d$ are the integers $6,7,8,9,10$ and none of these are coprime to 42 . Thus condition (17) is also satisfied. By Proposition 5.2, for each $1 \leq a \leq 41$ with $\operatorname{gcd}(a, 42)=1$ we have

$$
\left(\frac{a}{42} M-\frac{4471}{10500} \cdot K^{\frac{1}{3}}, \frac{a}{42} M-\frac{73}{210} \cdot K^{\frac{1}{3}}\right) \subseteq \operatorname{Bad}(\mathcal{W}) .
$$

One of the 729 intervals that make up $\operatorname{Bad}(\mathcal{W})$ is $[u, v)$ where the end points $u, v$ are

$$
\begin{aligned}
u= & 3895173640423584874713349032421520960246664873653293975537400307522458157015057896 \\
& 8661382487115397667257923729694373737120676906393201731077732461793807977510051609 \\
& 36231041460322490961793995991410145937421686204642056677472293123392066 \frac{3}{10}, \\
v= & 3895173640423584874713349032421520960246664873653293975537400307522458157015057896 \\
& 8661382487115397667257923729694373737120676906393201731077732461793807977510107869 \\
& 7615391607190077739469387928238665618669912989320140106379011502569660,
\end{aligned}
$$

and we checked that the interval in (25) with $a=1$ is contained in $[u, v)$. It is also interesting to note how close the two intervals are in length: the ratio of the lengths of the two intervals is

$$
\frac{\left(\frac{4471}{10500}-\frac{73}{210}\right) \cdot K^{\frac{1}{3}}}{v-u} \approx 0.9994
$$

which illustrates how remarkably accurate our Proposition 5.2 is.

\section{Completing the proof of Theorem 1}

It remains to apply Proposition 2.2 to the intervals $\left(\frac{9}{10}\right)^{n+1} \mathscr{K} \leq N \leq\left(\frac{9}{10}\right)^{n} \mathscr{K}$ with $3998 \leq n \leq 4226$. We write $K=K_{2}=\left(\frac{9}{10}\right)^{n} \mathscr{K}$ and $K_{1}=\left(\frac{9}{10}\right)^{n+1} \mathscr{K}$. It is no longer practical to use the choices in Lemma 2.3 as the interval in (9) is too short to contain many squarefree $m$ whose prime divisors are 3 and small primes $\equiv 5(\bmod 6)$. The interval in (9) is a result of imposing the uniform choices $\varepsilon_{m}=0$ and $\delta_{m}=\frac{1}{10}$. Instead we consider integers $m$ satisfying conditions (i)-(iii) of Section 2 but belonging to the (much larger) interval

$$
\frac{12}{5} K^{\frac{1}{3}} \leq m \leq \frac{16}{5} K^{\frac{1}{3}} .
$$

For each such $m$ we take $\varepsilon_{m}=\varepsilon^{\prime} / 1000$ and $\delta_{m}=\delta^{\prime} / 1000$ where $\varepsilon^{\prime}, \delta^{\prime}$ are integers with $\varepsilon^{\prime}$ and $\delta^{\prime}$ respectively as small and as large as possible such that the conditions (v), (vi) of Section 2 are satisfied. We only keep those values of $m$ for which

$$
0 \leq \varepsilon_{m}<\delta_{m} \leq 1 \quad \text { and } \quad \delta_{m}-\varepsilon_{m} \geq \frac{1}{20} ;
$$


an elementary though lengthy analysis in fact shows that the inequalities in (27) together with (v) and (vi) force $m$ to belong to the interval (26). Note that the set $\operatorname{Bad}\left(m, \varepsilon_{m}, \delta_{m}\right)$ has "relative density" $1-\delta_{m}+\varepsilon_{m}$ in $\mathbb{R}$; the restriction $\delta_{m}-\varepsilon_{m} \geq \frac{1}{20}$ ensures that this relative density is not too close to 1 , and that therefore $m$ makes a significant contribution to depleting the intervals in Algorithms 1 and 2.

We choose a prime $q \equiv 5(\bmod 6)$, depending on $K$, and let

$$
M_{0}=3 \cdot 5 \cdot 11 \cdots q,
$$

which is the product of 3 and the primes $\leq q$ that are $\equiv 5(\bmod 6)$. Let $\mathcal{W}_{0}$ be the set of positive integers dividing $M_{0}$ and satisfying the above conditions. We found experimentally that for each $n$ in the above range it is always possible to choose $q$ so that

$$
M_{0}=\operatorname{lcm}\left(\mathcal{W}_{0}\right), \quad \prod_{m \in \mathcal{W}_{0}}\left(1-\delta_{m}+\varepsilon_{m}\right) \leq \frac{1}{5}, \quad \text { and } \quad \log _{10}\left(M_{0} / K^{\frac{1}{3}}\right) \leq 7.5 .
$$

The inequality $\prod_{m \in \mathcal{W}_{0}}\left(1-\delta_{m}+\varepsilon_{m}\right) \leq \frac{1}{5}$ indicates that $\ell\left(\operatorname{Bad}\left(\mathcal{W}_{0}\right) \cap\left[0, M_{0}\right)\right)$ should heuristically be at most $M_{0} / 5$ which means that this is a good first step at depleting the interval $\left[0, M_{0}\right)$. The other inequality indicates that we can compute $\operatorname{Bad}\left(\mathcal{W}_{0}\right) \cap\left[0, M_{0}\right)$ in a reasonable number of steps, according to the heuristic, following Algorithm 1 . We let $p_{0}$ be the first prime $\equiv 5(\bmod 6)$ that is $>q$, and $p_{1}$ be the next such prime and so on. We let $M_{i+1}=p_{i} M_{i}$ and construct a tower as before. We stop once $\operatorname{Bad}\left(\mathcal{W}_{i}\right) \cap\left[0, M_{i}\right)$ satisfies the criterion of Proposition 2.2. Our Magma script succeeded in doing this for all $n$ in the range $3998 \leq n \leq 4226$. The total CPU time was around 2750 hours, but the computation was spread over 59 processors so the actual time was less than 2 days.

We give a few of details for the computation for the value $n=4226$. The final $M$ is the product of 3 and the primes $p \equiv 5(\bmod 6)$ that are $\leq 227$. The final $\mathcal{W}$ has 8083 elements. It turns out that $\operatorname{Bad}(\mathcal{W}) \cap[0, M)$ consists of 305 intervals and that $\ell(\operatorname{Bad}(\mathcal{W}) \cap[0, M)) / M \approx 2.24 \times 10^{-32}$.

\section{Acknowledgements}

The programs that accompany this paper are available at http://tinyurl.com/zlaeweo. It is a pleasure to thank Alex Bartel, Tim Browning, John Cremona, Roger HeathBrown and Trevor Wooley for stimulating discussions. We are grateful to the referee for a careful reading of the paper and for pointing out several corrections.

\section{References}

[Baer 1913] W. S. Baer, Beiträge zum Waringschen Problem, dissertation, University of Göttingen, 1913. $\mathrm{Zbl}$ 
[Bertault et al. 1999] F. Bertault, O. Ramaré, and P. Zimmermann, "On sums of seven cubes”, Math. Comp. 68:227 (1999), 1303-1310. MR Zbl

[Boklan and Elkies 2009] K. D. Boklan and N. D. Elkies, "Every multiple of 4 except 212, 364, 420, and 428 is the sum of seven cubes", preprint, 2009. arXiv

[Bosma et al. 1997] W. Bosma, J. Cannon, and C. Playoust, "The Magma algebra system, I: The user language", J. Symbolic Comput. 24:3-4 (1997), 235-265. MR Zbl

[Deshouillers et al. 2000] J.-M. Deshouillers, F. Hennecart, and B. Landreau, “7 $373170279850 ”$, Math. Comp. 69:229 (2000), 421-439. MR

[Dickson 1927] L. E. Dickson, “Extensions of Waring's Theorem on Nine Cubes”, Amer. Math. Monthly 34:4 (1927), 177-183. MR Zbl

[Dickson 1939] L. E. Dickson, "All integers except 23 and 239 are sums of eight cubes", Bull. Amer. Math. Soc. 45 (1939), 588-591. MR Zbl

[Elkies 2010] N. D. Elkies, "Every even number greater than 454 is the sum of seven cubes", preprint, 2010. arXiv

[Jacobi 1851] C. G. J. Jacobi, "Über die Zusammensetzung der Zahlen aus ganzen positiven Cuben; nebst einer Tabelle für die kleinste Cubenanzahl, aus welcher jede Zahl bis 12000 zusammengesetzt werden kann”, J. Reine Angew. Math. 42 (1851).

[Kempner 1912] A. Kempner, "Bemerkungen zum Waringschen Problem", Math. Ann. 72:3 (1912), 387-399. MR Zbl

[Landau 1908] E. Landau, "Über eine Anwendung der Primzahltheorie auf das Waringsche Problem in der elementaren Zahlentheorie", Math. Ann. 66:1 (1908), 102-105. MR

[Linnik 1943] U. V. Linnik, "On the representation of large numbers as sums of seven cubes", Mat. Sbornik (N.S.) 12(54):2 (1943), 218-224. MR Zbl

[Maillet 1895] E. Maillet, "Sur la décomposition d'un nombre entier en une somme de cubes d'entiers positifs”, Assoc. Franç. Bordeaux Notes Mém. 24 (1895), 242-247. JFM

[McCurley 1984] K. S. McCurley, "An effective seven cube theorem", J. Number Theory 19:2 (1984), 176-183. MR Zbl

[Ramaré 2005] O. Ramaré, “An explicit seven cube theorem”, Acta Arith. 118:4 (2005), 375-382. MR Zbl

[Ramaré 2007] O. Ramaré, "An explicit result of the sum of seven cubes", Manuscripta Math. 124:1 (2007), 59-75. MR Zbl

[Romani 1982] F. Romani, “Computations concerning Waring's problem for cubes”, Calcolo 19:4 (1982), 415-431. MR Zbl

[von Sterneck 1903] R. D. von Sterneck, "Über die kleinste Anzahl Kuben, aus welchen jede Zahl bis 40000 zusammengesetzt werden kann”, Akad. Wiss. Wien, Math.-Natur. Kl. Sitz. (IIa) 112 (1903), 1627-1666. Zbl

[Watson 1951] G. L. Watson, "A proof of the seven cube theorem", J. London Math. Soc. (2) 26 (1951), 153-156. MR Zbl

[Wieferich 1908] A. Wieferich, "Beweis des Satzes, daß sich eine jede ganze Zahl als Summe von höchstens neun positiven Kuben darstellen läßt”, Math. Ann. 66:1 (1908), 95-101. MR JFM

Communicated by Roger Heath-Brown

Received 2016-01-06 Revised 2016-08-21 Accepted 2016-09-23

samir.siksek@gmail.com Mathematics Institute, University of Warwick, Coventry, CV4 7AL, United Kingdom 


\section{Algebra \& Number Theory}

msp.org/ant

\section{EDITORS}

MANAGING EDITOR

Bjorn Poonen

Massachusetts Institute of Technology

Cambridge, USA

\author{
EDITORIAL BOARD CHAIR \\ David Eisenbud \\ University of California \\ Berkeley, USA
}

BOARD OF EDITORS

$\begin{aligned} \text { Dave Benson } & \text { University of Aberdeen, Scotland } & \text { Susan Montgomery } & \text { University of Southern California, USA } \\ \text { Richard E. Borcherds } & \text { University of California, Berkeley, USA } & \text { Shigefumi Mori } & \text { RIMS, Kyoto University, Japan } \\ \text { John H. Coates } & \text { University of Cambridge, UK } & \text { Raman Parimala } & \text { Emory University, USA } \\ \text { J-L. Colliot-Thélène } & \text { CNRS, Université Paris-Sud, France } & \text { Jonathan Pila } & \text { University of Oxford, UK } \\ \text { Brian D. Conrad } & \text { Stanford University, USA } & \text { Anand Pillay } & \text { University of Notre Dame, USA } \\ \text { Hélène Esnault } & \text { Freie Universität Berlin, Germany } & \text { Victor Reiner } & \text { University of Minnesota, USA } \\ \text { Hubert Flenner } & \text { Ruhr-Universität, Germany } & \text { Peter Sarnak } & \text { Princeton University, USA } \\ \text { Sergey Fomin } & \text { University of Michigan, USA } & \text { Joseph H. Silverman } & \text { Brown University, USA } \\ \text { Edward Frenkel } & \text { University of California, Berkeley, USA } & \text { Michael Singer } & \text { North Carolina State University, USA } \\ \text { Andrew Granville } & \text { Université de Montréal, Canada } & \text { Vasudevan Srinivas } & \text { Tata Inst. of Fund. Research, India } \\ \text { Joseph Gubeladze } & \text { San Francisco State University, USA } & \text { J. Toby Stafford } & \text { University of Michigan, USA } \\ \text { Roger Heath-Brown } & \text { Oxford University, UK } & \text { Ravi Vakil } & \text { Stanford University, USA } \\ \text { Craig Huneke } & \text { University of Virginia, USA } & \text { Michel van den Bergh } & \text { Hasselt University, Belgium } \\ \text { Kiran S. Kedlaya } & \text { Univ. of California, San Diego, USA } & \text { Marie-France Vignéras } & \text { Université Paris VII, France } \\ \text { János Kollár } & \text { Princeton University, USA } & \text { Kei-Ichi Watanabe } & \text { Nihon University, Japan } \\ \text { Yuri Manin } & \text { Northwestern University, USA } & \text { Efim Zelmanov } & \text { University of California, San Diego, USA } \\ \text { Philippe Michel } & \text { École Polytechnique Fédérale de Lausanne } & \text { Shou-Wu Zhang } & \text { Princeton University, USA }\end{aligned}$

PRODUCTION

production@msp.org

Silvio Levy, Scientific Editor

See inside back cover or msp.org/ant for submission instructions.

The subscription price for 2016 is US $\$ 290$ /year for the electronic version, and $\$ 485 /$ year (+\$55, if shipping outside the US) for print and electronic. Subscriptions, requests for back issues and changes of subscribers address should be sent to MSP.

Algebra \& Number Theory (ISSN 1944-7833 electronic, 1937-0652 printed) at Mathematical Sciences Publishers, 798 Evans Hall \#3840, c/o University of California, Berkeley, CA 94720-3840 is published continuously online. Periodical rate postage paid at Berkeley, CA 94704, and additional mailing offices.

ANT peer review and production are managed by EditFLow ${ }^{\circledR}$ from MSP.

\section{PUBLISHED BY}

- mathematical sciences publishers

nonprofit scientific publishing

http://msp.org/

() 2016 Mathematical Sciences Publishers 


\section{Algebra \& Number Theory}

Volume $10 \quad$ No. $10 \quad 2016$

Weight functions on Berkovich curves

MATTHEW BAKER and JOHANNES NICAISE

Nonvanishing of Dirichlet $L$-functions

2081

RIZWANUR KHAN and HIEU T. NGO

2093

Every integer greater than 454 is the sum of at most seven positive cubes

SAMIR SIKSEK

BERNARD LE STUM

JAN STEFFEn MÜlLER and Michael StOLL

BRUNO CHIARELLOTTO and CHRISTOPHER LAZDA

The Voronoi formula and double Dirichlet series

EREN MEHMET KiraL and FAN ZHOU

Pavel Etingof and Chelsea WALton 